\title{
PRECURSORS PRIOR TO TYPE IIn SUPERNOVA EXPLOSIONS ARE COMMON: PRECURSOR RATES, PROPERTIES, AND CORRELATIONS
}

\author{
Eran O. Ofek ${ }^{1}$, Mark Sullivan ${ }^{2}$, Nir J. Shaviv ${ }^{3}$, Aviram Steinbok ${ }^{1}$, Iair Arcavi ${ }^{1}$, Avishay Gal-Yam ${ }^{1}$, David Tal ${ }^{1}$, \\ Shrinivas R. Kulkarni ${ }^{4}$, Peter E. Nugent ${ }^{5,6}$, SAgi Ben-Ami $^{1}$, Mansi M. Kasliwal ${ }^{7},{\text { S. Bradley } \text { Cenko }^{8} \text {, Russ Laher }}^{9}$, \\ Jason Surace $^{9}$, Joshua S. Bloom ${ }^{6}$, Alexei V. Filippenko ${ }^{6}$, JefFrey M. Silverman $^{10}$, and Ofer Yaron ${ }^{1}$ \\ ${ }^{1}$ Benoziyo Center for Astrophysics, Weizmann Institute of Science, 76100 Rehovot, Israel \\ ${ }^{2}$ School of Physics and Astronomy, University of Southampton, Southampton SO17 1BJ, UK \\ ${ }^{3}$ Racah Institute of Physics, The Hebrew University, 91904 Jerusalem, Israel \\ ${ }^{4}$ Cahill Center for Astronomy and Astrophysics, California Institute of Technology, Pasadena, CA 91125, USA \\ ${ }^{5}$ Lawrence Berkeley National Laboratory, 1 Cyclotron Road, Berkeley, CA 94720, USA \\ ${ }^{6}$ Department of Astronomy, University of California, Berkeley, CA 94720-3411, USA \\ ${ }^{7}$ Observatories of the Carnegie Institution for Science, 813 Santa Barbara Street, Pasadena, CA 91101, USA \\ ${ }^{8}$ Astrophysics Science Division, NASA/Goddard Space Flight Center, Mail Code 661, Greenbelt, MD 20771, USA \\ ${ }^{9}$ Spitzer Science Center, California Institute of Technology, M/S 314-6, Pasadena, CA 91125, USA \\ ${ }^{10}$ Department of Astronomy, University of Texas, Austin, TX 78712, USA \\ Received 2014 January 21; accepted 2014 May 22; published 2014 June 19
}

\begin{abstract}
There is a growing number of Type IIn supernovae (SNe) which present an outburst prior to their presumably final explosion. These precursors may affect the SN display, and are likely related to poorly charted phenomena in the final stages of stellar evolution. By coadding Palomar Transient Factory (PTF) images taken prior to the explosion, here we present a search for precursors in a sample of 16 Type IIn SNe. We find five SNe IIn that likely have at least one possible precursor event (PTF 10bjb, SN 2010mc, PTF 10weh, SN 2011ht, and PTF 12cxj), three of which are reported here for the first time. For each SN we calculate the control time. We find that precursor events among SNe IIn are common: at the one-sided $99 \%$ confidence level, $>50 \%$ of SNe IIn have at least one pre-explosion outburst that is brighter than $3 \times 10^{7} L_{\odot}$ taking place up to $1 / 3 \mathrm{yr}$ prior to the $\mathrm{SN}$ explosion. The average rate of such precursor events during the year prior to the SN explosion is likely $\gtrsim 1 \mathrm{yr}^{-1}$, and fainter precursors are possibly even more common. Ignoring the two weakest precursors in our sample, the precursors rate we find is still on the order of one per year. We also find possible correlations between the integrated luminosity of the precursor and the SN total radiated energy, peak luminosity, and rise time. These correlations are expected if the precursors are mass-ejection events, and the early-time light curve of these $\mathrm{SNe}$ is powered by interaction of the SN shock and ejecta with optically thick circumstellar material.
\end{abstract}

Key words: stars: mass-loss - supernovae: general - supernovae: individual (SN 2010mc, PTF 10bjb, SN 2011ht, PTF 10weh, PTF 12cxj, SN 2009ip)

Online-only material: color figures, machine-readable tables

\section{INTRODUCTION}

In flux-limited synoptic surveys, a few percent of all discovered supernovae (SNe) show narrow- to intermediate-width $\left(\sim 30-3000 \mathrm{~km} \mathrm{~s}^{-1}\right)$ hydrogen and helium emission lines. These are dubbed Type IIn SNe (Schlegel 1990; Filippenko 1997; Kiewe et al. 2012) and they probably have massive progenitors (e.g., Gal-Yam \& Leonard 2009). Their emission lines likely originate from relatively abundant circumstellar material (CSM) around the SN progenitor star (e.g., Chevalier \& Fransson 1994; Chugai \& Danziger 1994; Chugai et al. 2003; Ofek et al. 2007, 2010, 2014b; Smith et al. 2008, 2009), ejected only a short time (on the order of months to decades) prior to the SN explosion (Dessart et al. 2009; Gal-Yam \& Leonard 2009; Ofek et al. 2010, 2013b). Several theoretical mechanisms have been suggested to explain this high mass loss in the final stages of stellar evolution (e.g., Rakavy et al. 1967; Woosley et al. 2007; Yoon \& Cantiello 2010; Arnett \& Meakin 2011; Chevalier 2012; Quataert \& Shiode 2012; Shiode \& Quataert 2014; Soker \& Kashi 2013).

Recently, five $\mathrm{SNe}$ with candidate pre-explosion outbursts (precursors) have been detected a few months to years prior to the SN explosion: SN 2006jc (Foley et al. 2007; Pastorello et al.
2007), SN 2009ip (Mauerhan et al. 2013a, 2014; Pastorello et al. 2013), SN 2010mc (PTF 10tel; Ofek et al. 2013b), PTF 11qcj (Corsi et al. 2014), and SN 2011ht (Fraser et al. 2013). In most cases, these precursors were detected from SNe IIn, or closely related events.

The frequency and properties of these precursors are critical for pinpointing the eruption mechanisms and understanding their effect on the eventual SN optical display, and may change our view of the final stages of massive star evolution. Therefore, we have conducted a search for precursor events in a sample of nearby SNe IIn for which we have pre-explosion observations from the Palomar Transient Factory ( $\mathrm{PTF}^{11}$; Law et al. 2009; Rau et al. 2009). Our sample contains 16 SNe IIn, and we found precursors events for five of the $\mathrm{SNe}$ in our sample. For the first time, we estimate the rate of such events and show that they are common. Furthermore, we investigate possible correlations between the properties of the precursors and the SNe. We note that some of the results presented in this paper, and in particular the suggested correlations, require confirmation based on larger samples. Such observations will likely be provided by future synoptic surveys.

\footnotetext{
11 http://ptf.caltech.edu/iptf/
} 
Table 1

SN Sample

\begin{tabular}{|c|c|c|c|c|c|c|c|c|c|}
\hline Name & Type & $\begin{array}{l}\alpha(\mathrm{J} 2000) \\
\quad(\mathrm{deg})\end{array}$ & $\begin{array}{c}\delta(\mathbf{J} 2000) \\
(\mathrm{deg})\end{array}$ & $\begin{array}{c}M_{R, \text { peak }} \\
\text { (mag) }\end{array}$ & $z$ & $\begin{array}{c}\mathrm{DM} \\
(\mathrm{mag})\end{array}$ & $\begin{array}{l}t_{\text {rise }} \\
\text { (day) }\end{array}$ & $\begin{array}{c}t_{\text {peak }} \\
\text { (day) }\end{array}$ & FAP \\
\hline SN 2010j1 & IIn & 145.722236 & +09.495037 & -20.6 & 0.011 & 33.44 & 55474 & 55494 & 0.01 \\
\hline SN 2010jj & IIn & 31.717743 & +44.571558 & $-18.0:$ & 0.016 & 34.22 & 55512 & 55525 & 0.02 \\
\hline PTF 10achk & IIn & 46.489751 & -10.522491 & -18.7 & 0.0327 & 35.77 & 55535 & 55550 & 0.00 \\
\hline PTF 10bjb & IIn? & 192.424667 & -10.800159 & $<-16.4$ & 0.026 & 35.27 & 55326 & 55434 & \\
\hline SN 2010bq & IIn & 251.730659 & +34.159641 & -18.5 & 0.032 & 35.73 & 55296 & 55311 & 0.00 \\
\hline PTF 10gvf & IIn & 168.438496 & +53.629126 & -18.8 & 0.081 & 37.82 & 55321 & 55339 & 0.00 \\
\hline SN $2010 \mathrm{mc}$ & IIn & 260.377817 & +48.129834 & -18.5 & 0.035 & 35.93 & 55427 & 55447 & 0.00 \\
\hline PTF 10weh & IIn & 261.710251 & +58.852064 & -20.7 & 0.138 & 39.06 & 55453 & 55504 & 0.02 \\
\hline PTF 11fzz & IIn & 167.694502 & +54.105220 & -20.3 & 0.082 & 37.85 & 55721 & 55783 & 0.00 \\
\hline PTF $12 \mathrm{cxj}$ & IIn? & 198.161181 & +46.485090 & -17.3 & 0.036 & 35.96 & 56033 & 56048 & 0.01 \\
\hline SN 2011cc & IIn & 248.456000 & +39.263528 & $-18.1:$ & 0.0319 & 35.72 & 55624 & 55741 & 0.02 \\
\hline SN 2011fx & IIn & 4.498167 & +24.562778 & $-17.1:$ & 0.0193 & 34.61 & 55780: & 55803: & 0.00 \\
\hline SN 2011ht & IIn & 152.044083 & +51.849194 & -16.8 & 0.004 & 30.96 & 55830: & 55880: & 0.00 \\
\hline SN 2011hw & $\mathrm{IIn} / \mathrm{Ibn}$ & 336.560583 & +34.216417 & -19.1: & 0.023 & 34.99 & 55860 & 55884: & $\cdots$ \\
\hline SN 2011iw & IIn & 353.700833 & -24.750444 & $-18.1:$ & 0.023 & 34.99 & 55819 & 55894: & 0.04 \\
\hline SN 2012as & IIn & 231.285500 & +37.963722 & $-18.0:$ & 0.0297 & 35.56 & 55968: & 55976: & 0.00 \\
\hline
\end{tabular}

Notes. The SN sample. Type refers to SN classification; $\alpha(\mathrm{J} 2000)$ and $\delta(\mathrm{J} 2000)$ are the $\mathbf{J} 2000.0$ right ascension and declination, respectively. $M_{R \text {,peak }}$ is the peak absolute $R$-band magnitude, $z$ is the SN redshift, DM is distance modulus, $t_{\text {rise }}$ is the MJD of the estimated start of the SN rise, and $t_{\text {peak }}$ is the MJD of the light-curve peak. Colon indicates an uncertain value. The $t_{\text {rise }}$ for SN 2011iw is based on a detection in coadded PTF data and it appears as part of the fast rise following this detection. FAP is the false-alarm probability to detect a precursor by coadding images in 15 day bins as estimated using the bootstrap method (see Section 4.2). The values are based on 100 bootstrap simulations and are therefore truncated to two figures after the decimal point. SNe with no data are those in which the precursor is clearly detected in many individual images and thus the bootstrap analysis on the coadded data is ineffective (see Section 4.2). SNe below the horizontal line were not triggered by PTF, although PTF has pre-explosion images of their locations.

References. SN 2010j1: PTF 10aaxf; Zhang et al. (2012), Newton \& Puckett (2010), Stoll et al. (2011), Smith et al. (2011), Chandra et al. (2012), Ofek et al. (2013a, 2014b), Moriya et al. (2014). SN 2010jj: PTF 10aazn; Rich (2010), Silverman et al. (2010). PTF 10achk: reported here for the first time. PTF 10bjb: reported here for the first time. SN 2010bq: PTF 10fjh; Duszanowicz (2010), Challis et al. (2010), Ofek et al. (2013a). PTF 10gvf: reported here for the first time. PTF 10tel: Ofek (2012), Ofek et al. (2013a; 2013b). PTF 10weh: reported here for the first time. PTF 11fzz: reported here for the first time. PTF 12cxj: reported here for the first time. SN 2011cc: Mason et al. (2011). SN 2011fx: Ciabattari et al. (2011). SN 2011ht: Prieto et al. (2011), Roming et al. (2012), Mauerhan et al. (2013b). SN 2011hw: Dintinjana et al. (2011), Smith et al. (2012). SN 2011iw: Mahabal et al. (2011). SN 2012as: Jin et al. (2012).

The paper is organized as follows. We describe the SN sample in Section 2, while the observations are presented in Section 3. The methodology of precursor selection is discussed in Section 4 and our candidates are presented in Section 5. We give our control-time estimate in Section 6 and the precursor rate in Section 7. The CSM mass estimate is discussed in Section 8, the correlations between the $\mathrm{SNe}$ and precursor properties in Section 9, and we conclude in Section 10.

\section{SAMPLE}

Our sample is based on SNe IIn that show intermediate-width Balmer emission lines. Inspecting hundreds of core-collapse $\mathrm{SNe}$ spectra obtained by PTF, we find that some SNe show this hallmark of SNe IIn at early times (a few days after the explosion), but these lines disappear on a timescale of a week (Gal-Yam et al. 2014; O. Yaron et al. 2014, in preparation). It is possible that these $\mathrm{SNe}$ also suffer from a moderate massloss rate prior to the explosion. We exclude from our sample objects for which the spectra evolve into those of normal SNe II a few weeks after explosion. Examples for such objects include PTF 11iqb and PTF 10uls (Ofek et al. 2013a). In total we rejected five such $\mathrm{SNe}$ from our sample. A search for precursors among these "weak" SNe IIn will be published elsewhere.

Another important criterion for our SNe selection is that they have a large number of pre-explosion images from the PTF survey. This is critical, as precursors may be relatively faint and it is desirable to coadd images in order to obtain a limiting magnitude that is deeper than that of the nominal survey. The
SNe in our sample were found by the PTF as well as by amateur astronomers, the Lick Observatory SN Search (Li et al. 2000; Filippenko et al. 2001), and the Catalina Real-time Transient Survey (Drake et al. 2009). We selected only nearby SNe, found within $400 \mathrm{Mpc}$, for which we have tens of pre-explosion observations. Table 1 list the $16 \mathrm{SNe}$ in our sample.

\section{OBSERVATIONS}

We used PTF observations of the SNe in our sample. The PTF data reduction is described by Laher et al. (2014), and the photometric calibration is discussed by Ofek et al. (2012a, $2012 b$ ). Our search is based on image subtraction, and the flux residuals in the individual image subtractions for all the SNe in our sample are listed in Table 2.

Figures 1 and 2 show the light curves before explosion (first and third columns) and after explosion (second and fourth columns) of all the SNe in our sample. The pre-explosion light curve shows the median flux, relative to the reference image flux, in 15 day bins. Only bins containing $\geqslant 6$ measurements are presented. The plus $(+)$ signs show the lower and upper $5 \sigma$ uncertainty relative to the reference image, while the solid lines connect consecutive bins. The uncertainties where calculated using the bootstrap error on the mean. The vertical dashed lines show the estimated explosion time and time of maximum light (see Table 1).

We obtained spectra of our SNe using various telescopes, and the log of selected observations is presented in Table 3. Some of the spectra are presented in this paper, while the rest are 
Table 2

SN Observations

\begin{tabular}{|c|c|c|c|c|c|c|c|c|}
\hline Name & Band & $\begin{array}{c}\text { MJD- } t_{\text {rise }} \\
\text { (day) }\end{array}$ & $\begin{array}{l}\text { MJD } \\
\text { (day) }\end{array}$ & $\begin{array}{l}\text { Mag } \\
\text { (mag) }\end{array}$ & $\begin{array}{l}\text { Mag Err } \\
(\mathrm{mag})\end{array}$ & $\begin{array}{c}\text { Lim Mag } \\
(\mathrm{mag})\end{array}$ & $\begin{array}{c}\text { Flux } \\
\text { (counts) }\end{array}$ & $\begin{array}{l}\text { Flux Err } \\
\text { (counts) }\end{array}$ \\
\hline PTF10aaxf & $R$ & -232.84900 & 55241.15100 & 81.555 & -0.913 & 21.441 & -66.4 & 55.8 \\
\hline PTF10aaxf & $R$ & -232.49900 & 55241.50100 & 82.323 & -0.985 & 20.590 & -134.7 & 122.2 \\
\hline PTF10aaxf & $R$ & -229.61100 & 55244.38900 & 21.890 & 0.392 & 21.802 & 110.7 & 40.0 \\
\hline PTF10aaxf & $R$ & -229.56800 & 55244.43200 & 82.112 & -0.653 & 21.247 & -110.9 & 66.7 \\
\hline PTF10aaxf & $R$ & -223.68700 & 55250.31300 & 81.913 & -1.045 & 20.936 & -92.3 & 88.8 \\
\hline
\end{tabular}

Notes. Photometric measurements and flux residuals of the SNe in our sample. Magnitude are calculated in luptitudes, which is a magnitude measure in which the logarithm is replaced with a hyperbolic sine. Unlike normal magnitudes, luptitudes are defined for negative fluxes (Lupton et al. 1999). and they have meaning only when smaller than the limiting magnitude.

(This table is available in its entirety in a machine-readable form in the online journal. A portion is shown here for guidance regarding its form and content.)

Table 3

Log of Spectroscopic Observations

\begin{tabular}{lccc}
\hline \hline SN Name & $\begin{array}{l}\text { MJD } \\
(\text { day })\end{array}$ & Telescope & Instrument \\
\hline SN 2010j1 & 55505 & Keck-I & LRIS \\
SN 2010jj & 55505 & Keck-I & LRIS \\
& 55544 & Lick 3 m & Kast \\
PTF 10achk & 55547 & UH88 & SNIFS \\
PTF 10bjb & 55262 & Keck-I & LRIS \\
SN 2010bq & 55300 & Gemini-N & GMOS \\
PTF 10gvf & 55322 & Keck-I & LRIS \\
SN 2010mc & 55434 & Gemini-N & GMOS \\
& 55442 & Lick 3 m & Kast \\
& 55449 & KPNO 4 m & RC Spec \\
& 55455 & Lick 3 m & Kast \\
PTF 10weh & 55479 & KPNO 4 m & RC Spec \\
& 55502 & Lick 3 m & Kast \\
& 55503 & KPNO 4 m & RC Spec \\
PTF 11fzz & 55736 & Hale 5 m & DBSP \\
& 55873 & WHT & ISIS \\
PTF 12cxj & 56035 & Gemini-N & GMOS \\
\hline
\end{tabular}

Note. MJD is the observation modified Julian day.

available electronically from the WISeREP Web site ${ }^{12}$ (Yaron \& Gal-Yam 2012).

\section{PRECURSOR CANDIDATE SELECTION}

In this section we describe the methods we used to find the precursor candidates. In Section 4.1 we present the search methods, while in Section 4.2 we discuss the reliability of our methodology and the false-alarm probability of the precursor candidates.

\subsection{Detection Methods}

Our candidate precursor selection is based on two channels. The first channel identifies precursors that were detected in a single image prior to the SN explosion, at the $6 \sigma$ level, without the need to coadd data. The second channel identifies precursors that were detected in the coadded data at the $5 \sigma$ level, using 15 day bins and including only bins that contain more than five flux measurements.

The second channel significantly increases the sensitivity of PTF to precursor events by effectively coadding images of a SN location in time bins of 15 days. These time bins often include

\footnotetext{
12 http://www.weizmann.ac.il/astrophysics/wiserep/
}

a large number of observations, extending to depths beyond the nominal PTF survey limiting magnitude, and reaching an $R$-band limiting magnitude of $<23.5$. However, coadding images themselves in arbitrary time bins, and then conducting image-subtraction analysis on each trial bin, is computationally expensive. Instead, we carefully apply image subtraction to individual images, and for each image we save the flux residual (negative or positive) at the location of the SN. We can then coadd the scalar flux residuals in any temporal combination we desire. This method was used in the case of SN 2010mc (PTF 10tel; Ofek et al. 2013b) and PTF 11qcj (Corsi et al. 2014).

In the first channel, the uncertainty (i.e., $\sigma$ ) was estimated based on the Poisson noise propagated through the imagesubtraction pipeline, while for the second channel, we calculated uncertainties in each bin using the bootstrap technique (Efron 1982). We note that in most cases the bootstrap errors are consistent with the uncertainties derived based on the standard deviation of the points in each bin, and the expected Poisson noise. Therefore, our bootstrap error estimate suggests that the statistical uncertainties produced by the subtraction pipeline are realistic.

In order to keep our search uniform we use only the PTF $R$-band data for our search and analysis, as PTF was primarily an $R$-band search. However, we also show the $g$-band data in the various plots where available; the amount of $g$-band data is small in comparison to the $R$-band.

Candidate precursor events detected via one of the channels are discussed in Section 5. In the initial search we do not attempt to use different time bins. This decision was made in order to limit the number of statistical experiments, which may affect the significance of our results. We note, however, that careful examination of specific events with longer time bins may contain precursor events that are not discussed here. For example, in Corsi et al. (2014) we report on a possible faint (absolute magnitude -13), several months long, brightening in the light curve of the Type Ic PTF 11qcj about $2.5 \mathrm{yr}$ prior to its explosion.

We note that several objects show points that are marginally below the lower $5 \sigma$ error threshold. It is possible that this is caused by real variability of the progenitor (e.g., Szczygieł et al. 2012), but here we concentrate on the outbursts rather than possible dimmings (see also Section 5.6).

$$
\text { 4.2. Tests }
$$

We performed several tests to verify the reliability of our methodology, especially against false alarms. In order to test 

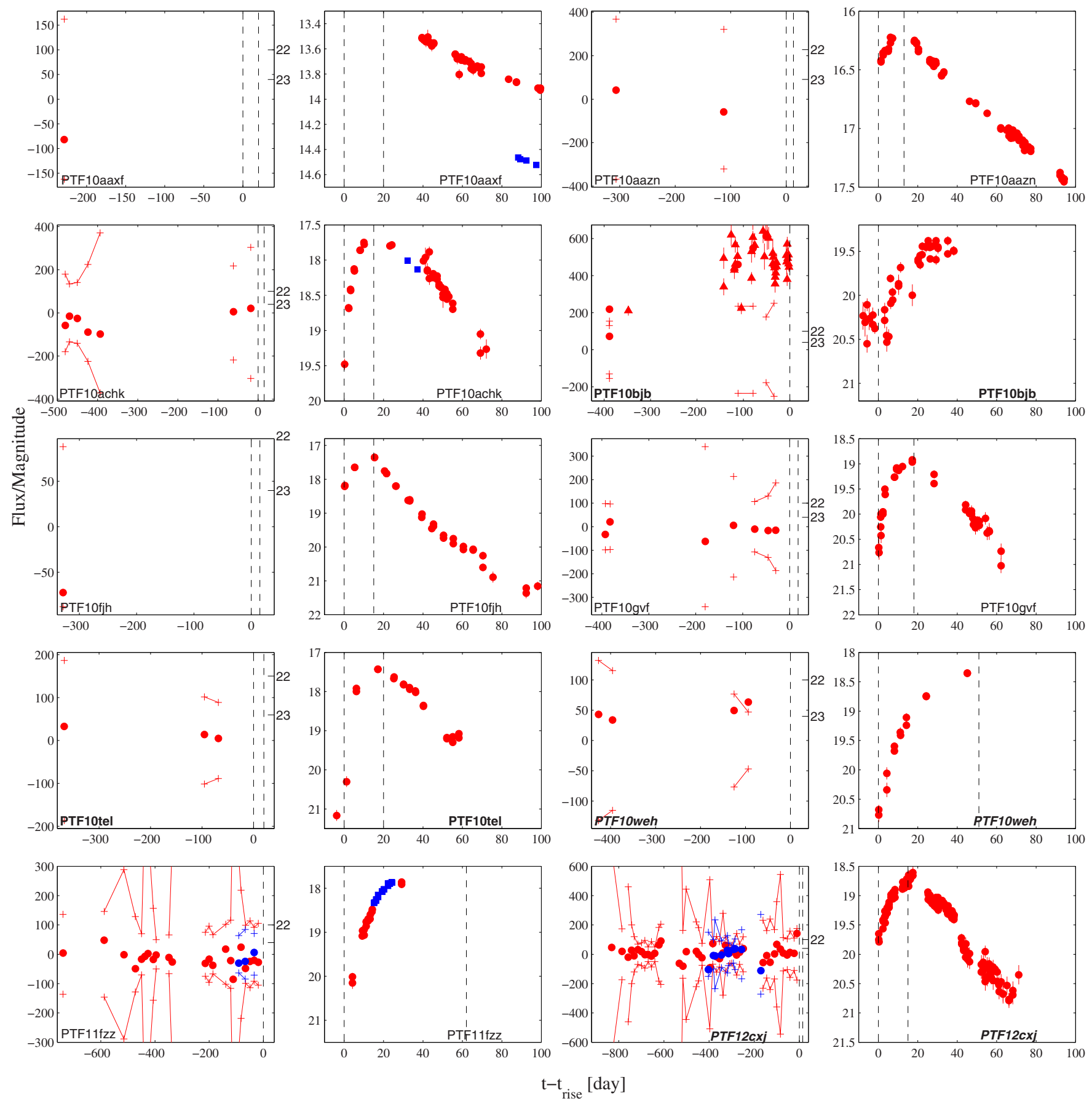

Figure 1. Light curves of $10 \mathrm{SNe}$ (names are listed in each panel). For each SN two plots are shown (side by side in the first and second columns and in the third and fourth columns). Columns two and four show the light curve after the SN explosion, while the panels in columns one and three give only the coadded flux residual (relative to the reference image) prior to the $\mathrm{SN}$ explosion (if there are more than five observations per time bin). The two vertical dashed lines show the assumed explosion date $\left(t_{\text {rise }}\right)$ and rough maximum-luminosity date ( $t_{\text {peak }}$; listed in Table 1$)$. Time is measured relative to $t_{\text {rise }}$. The red circles represent $R$-band observations while the blue squares show $g$-band data. All of the measurements are in the PTF magnitude system (Ofek et al. 2012a, 2012b). The zero point of the flux residuals (first and third columns) is 27, with the exception of PTF 10aazn (27.895), PTF 10bjb (27.14), and PTF 10tel (27.442). The tick marks on the right-hand axes of the first and third columns show the fluxes that correspond to PTF magnitudes of 22 and 23. In the first and third columns, filled symbols show the flux measurements in 15 day bins. The plus (+) signs represent the $5 \sigma$ upper and lower limits, as estimated from the bootstrap technique in each bin (Efron 1982). If plus signs are found in consecutive bins they are connected by a solid line. SN names marked in boldface are events which show precursors, while italics mark SNe with possible precursors.

(A color version of this figure is available in the online journal.)

detections made by the first channel (i.e., precursors detected in single images), we extracted the light curves at random positions on top of the same host galaxy, but shifted in position relative to the SN. We found that typically the probability to get a $6 \sigma$ detection is less than $0.1 \%$ per image. This is because the noise is not distributed normally (e.g., there are outliers) owing to occasional subtraction artifacts, cosmic rays, or asteroids in the field of view. For some SNe we have hundreds of images, and therefore the probability for a detection is on the order of a few percent per SN. Therefore, we consider as good candidates only events which have two consecutive detections (see Section 5). Figure 3 illustrates the histogram of "random"-position flux 

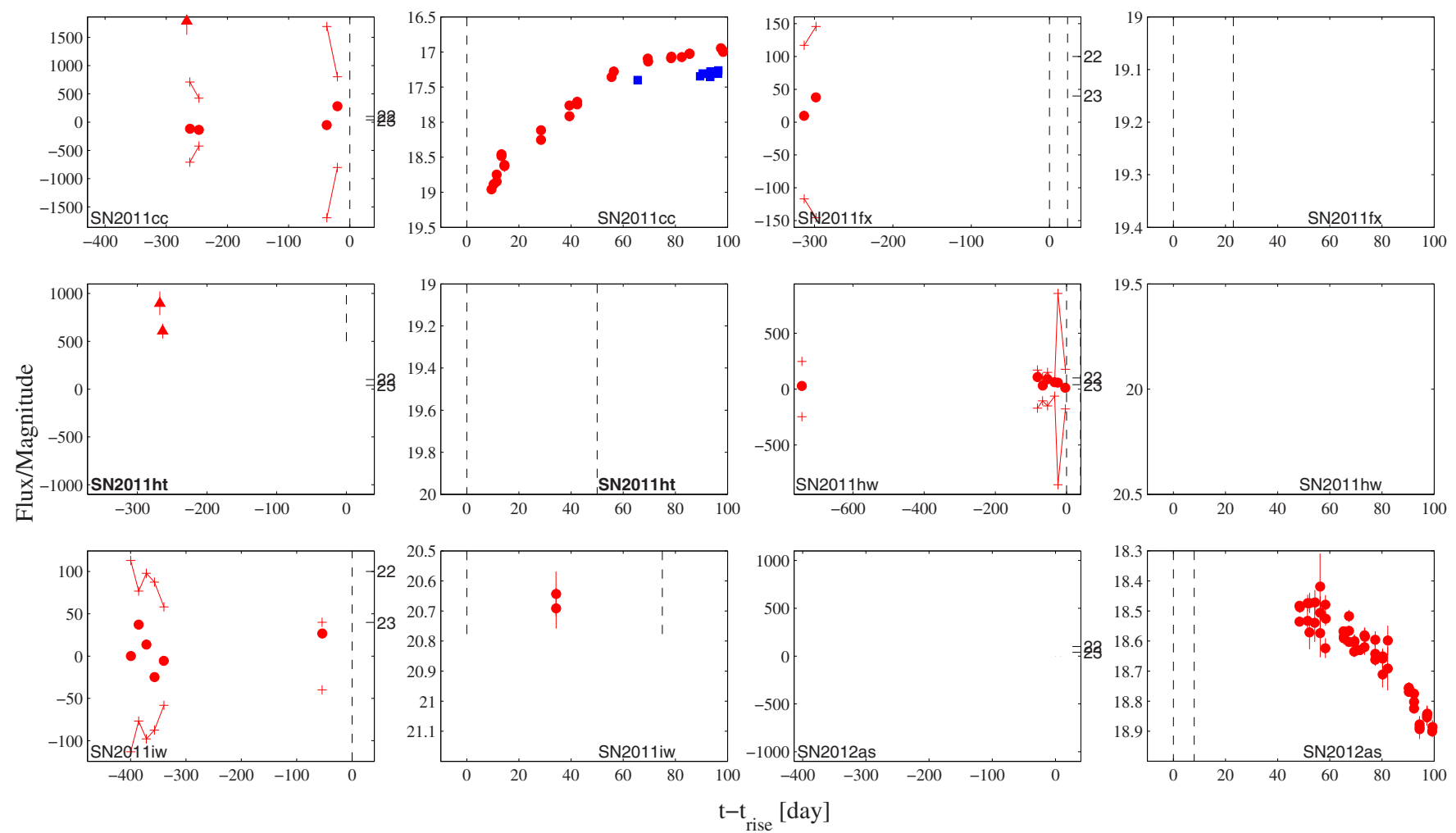

Figure 2. As in Figure 1 but for six additional SNe.

(A color version of this figure is available in the online journal.)

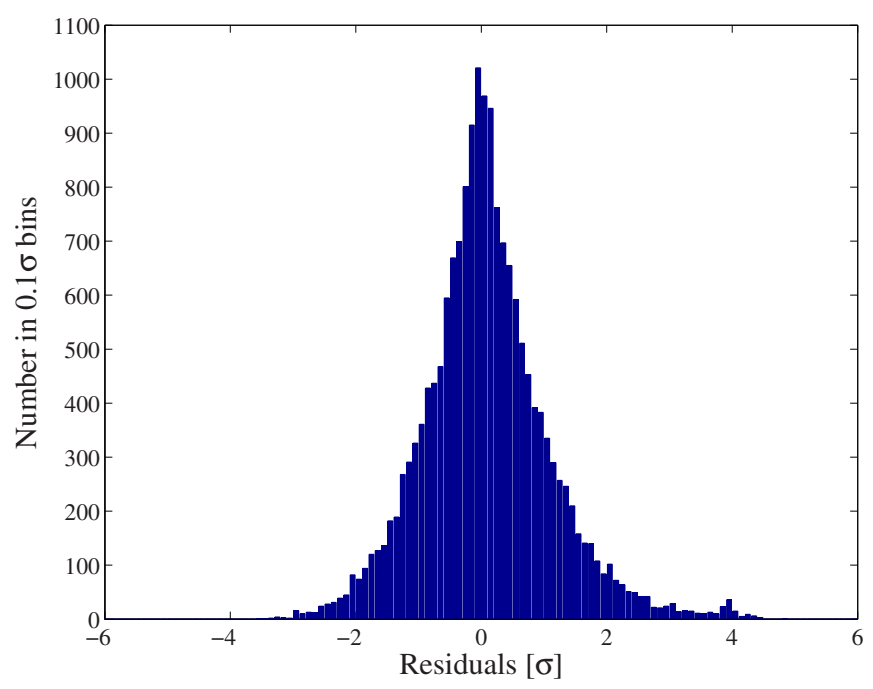

Figure 3. Distribution of flux residuals in $\sigma$ units, at all the epochs and at 16 random positions around the position of PTF 12cxj, in the same host galaxy.

(A color version of this figure is available in the online journal.)

residuals in units of the flux-residual errors (i.e., $\sigma$ ) for the case of random positions around PTF $12 \mathrm{cxj}$. It is apparent that even though there is a small excess around positive residuals, the probability of getting residuals exceeding $6 \sigma$ is small.

In order to test detections made using the second channel (coadding flux residuals in 15 day time bins), for each $\mathrm{SN}$ we run 100 bootstrap simulations in which we mixed the flux residuals and times, and binned the data again in 15 day bins with the same selection criteria. The probability to have a single detection, based on these simulations, is listed in column FAP (false-alarm probability) in Table 1 . This probability may approach several percent for the entire sequence of images. However, after passing the selection criteria, each candidate is tested using various binning schemes, and only sources that show two consecutive independent detections are considered to be good candidates (see Section 5).

We also search for correlations between the flux residuals and airmass, and flux residuals and the amplitude of the expected atmospheric refraction. Indeed, we find marginally significant correlations between these properties; however, they are too small to affect our results.

To summarize, tests of our methodology suggest that the false-alarm probability for a single-point precursor detection, in an entire SN data set, is $\sim 5 \%$ per object. In order to avoid false detections, we consider as precursor candidates only cases which show at least two detections clustered in time (i.e., assuming the noise is not correlated).

\section{CANDIDATE PRECURSOR EVENTS}

Our search yielded several SNe with candidate precursors. The precursors of PTF 10bjb, SN 2010mc, and SN 2011ht were clearly detected in individual images (first channel), while PTF 12cxj and PTF 10weh are weakly detected in coadded images (second channel). Although these two weak events pass all of our tests and we consider them to be real, given the low signal-to-noise ratio of the detections we also discuss our results assuming these two events are not real. Images of all the precursors are presented in Figure 4.

\subsection{PTF 10tel}

PTF 10tel is discussed in detail by Ofek et al. (2013b). For completeness, here we summarize the main properties of 

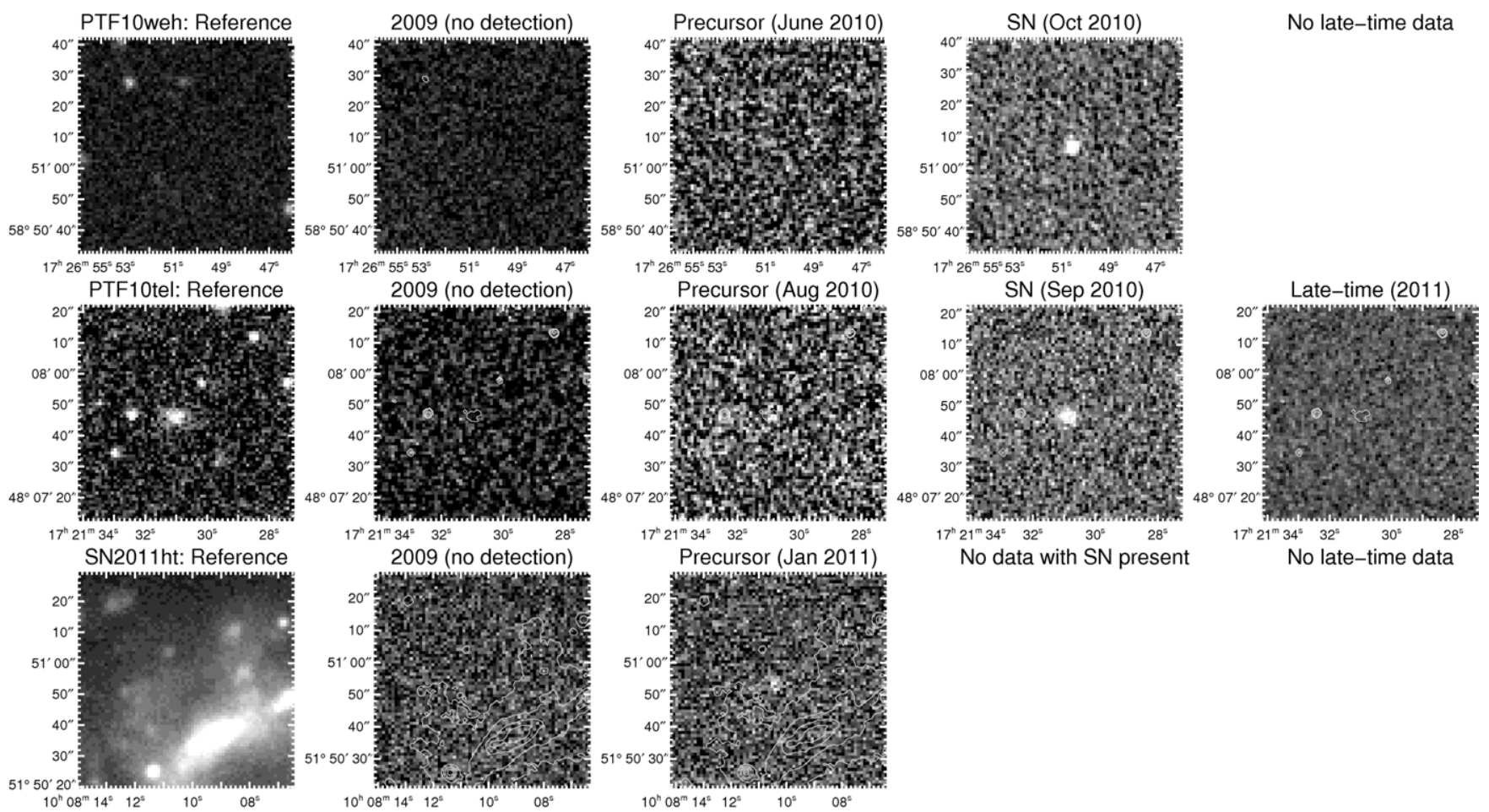

No data with SN present

No late-time data
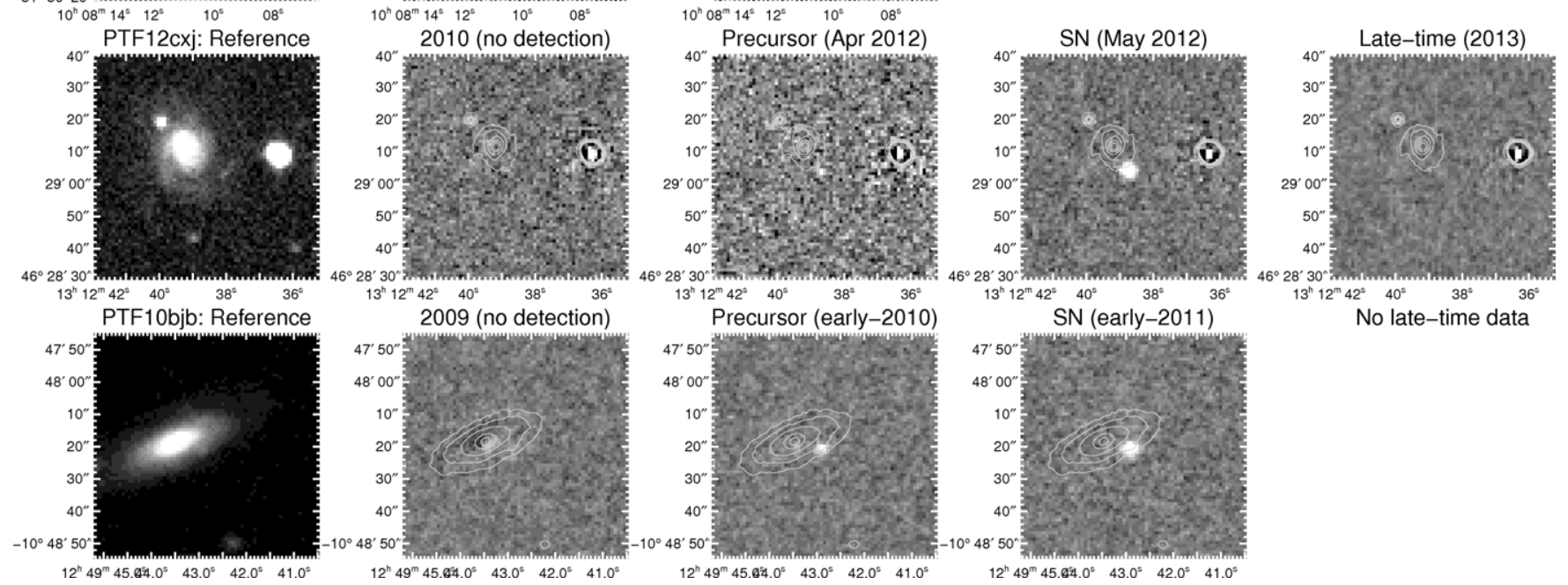

No late-time data

Figure 4. Images of the precursors, SN, and nondetections for the SNe PTF 10weh, PTF 10tel, SN $2011 \mathrm{ht}$, PTF 12cxj, and PTF 10bjb (from top to bottom). In each row, the five images present (left to right) the reference image, the pre-SN explosion nondetection, the brightest precursor for each object (where more than one was detected), the SN, and a post-SN image (if available). The contours from the reference image are also overplotted. All of the precursors can be clearly seen, except PTF 10weh, which is marginally detected. A detailed inspection of the images of PTF 10weh in which the precursor is detected do not reveal any cosmetic problems (e.g., cosmic rays) near the precursor location, and the balance of evidence is that this precursor is real (see discussion regarding false-alarm probability in Section 4.2). However, in Figure 19 we also show the correlations between the SN and precursor properties, excluding PTF 10weh.

this event. The observations of PTF 10tel show an outburst about 40 days prior to the probable explosion. This precursor is detected via both the first and second channels. Our photometric and spectroscopic observations suggest that it is produced by an energetic outburst releasing $\sim 10^{-2} M_{\odot}$ at typical velocities ${ }^{13}$ of $2000 \mathrm{~km} \mathrm{~s}^{-1}$, and powered by at least $6 \times 10^{47} \mathrm{erg}$ of energy.

For completeness we show in Figure 5 the light curve of PTF 10tel from Ofek et al. (2013b). The physical parameters of this $\mathrm{SN}$ and precursor are listed in Table 4.

\subsection{SN $2011 h t$}

SN 2011ht was discovered on 2011 September 29.2 (UTC dates are used throughout this paper), at apparent magnitude

13 This is the larger $\sigma$ of a two-component Gaussian fit.
17.0. Based on a spectrum obtained on 2011 September 30, Pastorello et al. (2011) suggested that it is an SN impostor sharing some similarities with the eruption of the luminous blue variable UGC 2773-OT (Smith et al. 2010). The spectrum shows narrow lines of $\mathrm{H}, \mathrm{Ca}$ II $(\mathrm{H} \& \mathrm{~K}$ and the near-infrared triplet, with P-Cygni profiles), and a forest of narrow Fe II absorption features. Also, prominent $\mathrm{NaI} \mathrm{D}, \mathrm{Sc}$ II, and $\mathrm{Ba}$ II features are detected in absorption. Prieto et al. (2011) obtained a later spectrum on 2011 November 11.5. They reported substantial evolution with respect to the initial classification, with strong Balmer and weaker $\mathrm{He}$ I and Fe II emission lines superposed on a blue continuum. Based on the spectrum and the SN absolute magnitude, they suggested that it is likely an SN IIn. This is supported by Roming et al. (2012), who reported on the Swift-UVOT observations of this SN, detecting a 7 mag rise 
Table 4

Precursor Properties

\begin{tabular}{|c|c|c|c|c|c|c|c|c|c|}
\hline Name & $\begin{array}{c}\Delta t \\
\text { (day) }\end{array}$ & $\begin{array}{c}\delta t \\
\text { (day) }\end{array}$ & $\begin{array}{l}L_{\mathrm{SN}, \text { peak }} \\
\left(\mathrm{erg} \mathrm{s}^{-1}\right)\end{array}$ & $\begin{array}{l}E_{\mathrm{SN}} \\
(\mathrm{erg})\end{array}$ & $\begin{array}{l}L_{\text {prec,peak }} \\
\left(\operatorname{erg~s}^{-1}\right)\end{array}$ & $\begin{array}{l}E_{\text {prec }} \\
\text { (erg) }\end{array}$ & $\begin{array}{l}\mathrm{H} \alpha_{\text {narrow }} \\
\left(\mathrm{km} \mathrm{s}^{-1}\right)\end{array}$ & $\begin{array}{c}\mathrm{H} \alpha_{\text {broad }} \\
\left(\mathrm{km} \mathrm{s}^{-1}\right)\end{array}$ & $\begin{array}{c}\epsilon^{-1} M_{\mathrm{CSM}} \\
\left(M_{\odot}\right)\end{array}$ \\
\hline $\mathrm{SN} 2010 \mathrm{mc}$ & -20 & 30 & $6.9 \times 10^{42}$ & $2 \times 10^{49}$ & $2.6 \times 10^{41}$ & $6 \times 10^{47}$ & $\sim 500$ & $\sim 2000$ & 0.015 \\
\hline PTF 10bjb & -85 & 110 & $>8 \times 10^{41}$ & $>2.0 \times 10^{49}$ & $3.3 \times 10^{41}$ & $2.4 \times 10^{48}$ & $\sim 300$ & $\sim 600$ & 0.67 \\
\hline SN $2011 \mathrm{ht}$ & -205 & 210 & $1.5 \times 10^{42}$ & $2.5 \times 10^{49}$ & $1.5 \times 10^{40}$ & $2 \times 10^{47}$ & 260 & 1800 & 0.006 \\
\hline PTF 10weh & -80 & 40 & $5.3 \times 10^{43}$ & $7.2 \times 10^{50}$ & $1.9 \times 10^{42}$ & $5 \times 10^{48}$ & $\sim 100$ & $\sim 1000$ & 0.5 \\
\hline PTF $12 \mathrm{cxj}-\mathrm{A}$ & -10 & 7 & $2.3 \times 10^{42}$ & $8 \times 10^{48}$ & $2.2 \times 10^{41}$ & $9 \times 10^{46}$ & $\sim 200$ & $\sim 1200$ & 0.006 \\
\hline PTF $12 c x j-B$ & -700 & 15 & & & $4.3 \times 10^{40}$ & $6 \times 10^{46}$ & & & 0.004 \\
\hline \multirow[t]{4}{*}{ SN 2009ip } & -25 & 50 & $4.6 \times 10^{42}$ & $3 \times 10^{49}$ & $2 \times 10^{41}$ & $8 \times 10^{47}$ & $\sim 200$ & $\sim 2000$ & 0.02 \\
\hline & -660 & $\ldots$ & & & $1 \times 10^{41}$ & $\ldots$ & & & 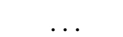 \\
\hline & -710 & 60 & & & $1 \times 10^{41}$ & $6 \times 10^{47}$ & & & 0.02 \\
\hline & -1060 & 60 & & & $2 \times 10^{41}$ & $6 \times 10^{47}$ & & & 0.02 \\
\hline
\end{tabular}

Notes. Properties of the precursors and $\mathrm{SNe}$. $\mathrm{H} \alpha_{\text {narrow }}$ and $\mathrm{H} \alpha_{\text {broad }}$ are the line width of the narrow and broad components of the $\mathrm{H} \alpha$ lines in units of the Gaussian $\sigma$ (multiply by 2.35 to get the FWHM). $\Delta t$ is the average time of the precursor. All of the values are order-of-magnitude estimates. The H $\alpha$ line widths for SN 2011ht are adopted from Roming et al. (2012). The values listed for SN 2009ip are adopted from Foley et al. (2011), Smith et al. (2010), Drake et al. (2010), Ofek et al. (2013c), and Margutti et al. (2014). SNe listed above the horizontal line are in our sample and are used to estimate the precursor rates. Events below the horizontal line are not in our sample, but are used in the correlation analysis (Figures 19 and 20). We note that SN 2006jc (Pastorello et al. 2007; Foley et al. 2007) was excluded from the correlation analysis since the CSM in this SN is hydrogen deficient.

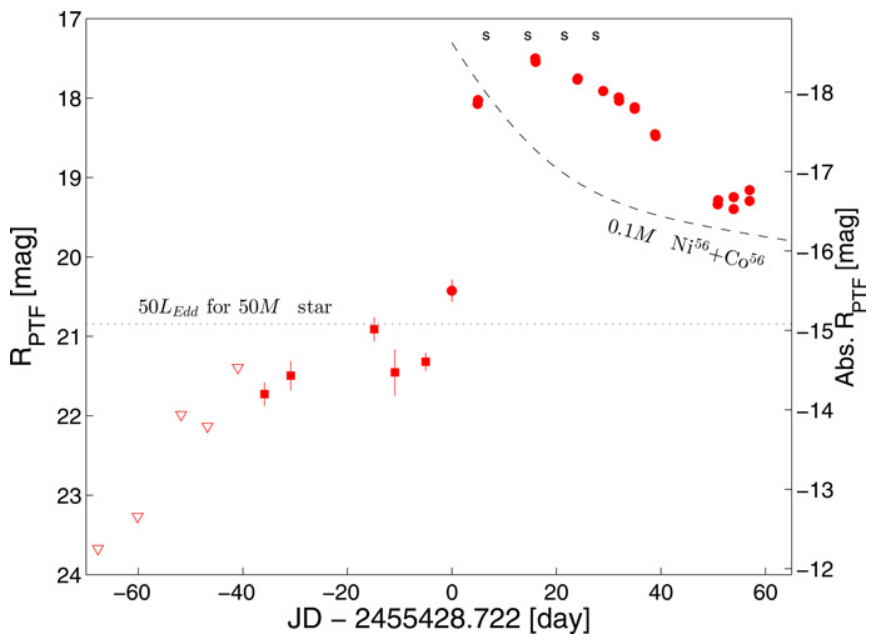

Figure 5. Light curve of SN 2010mc (PTF 10tel) as obtained with the Palomar 48 inch telescope. The red circles are based on individual images, the squares are based on coadded images, while the empty triangles represent the $3 \sigma$ upper limits derived from coadded images. The error bars represent the $1 \sigma$ uncertainty. The object magnitudes are given in the PTF magnitude system. The dashed line shows the expected luminosity from the radioactive decay of an ejected mass of $0.1 M_{\odot}$ of $\mathrm{Ni}^{56}$, assuming that at late times the optical depth is sufficiently large to convert the radioactive energy to optical luminosity, but not so large that it goes into $P d V$ work. This line represents an upper limit on the total amount of $\mathrm{Ni}^{56}$ in the ejecta; it was set to coincide with the latest observation of the $\mathrm{SN}$ at $\mathrm{JD} \approx 2,455,758$. The dotted line represents a bolometric luminosity equal to 50 times the Eddington luminosity for a $50 M_{\odot}$ star (an order-of-magnitude estimate of the mass of the progenitor assuming it is a massive star). The right edges of the "S" symbols above the light curve indicate the epochs at which we obtained spectra (figure adopted from Ofek et al. 2013b).

(A color version of this figure is available in the online journal.)

in the $U V W 2$ band over 40 days, peaking at a $u$-band magnitude of about -18 .

Fraser et al. (2013) reported on an outburst peaking at a $z$-band absolute magnitude of -11.8 detected $\sim 9$ months prior to the explosion, and with the last detection $\sim 4$ months before the explosion. The event was detected by both PanSTARRS-1 and the Catalina Sky Survey. The duration of the outburst is not well constrained, and it can be either a single event that lasted for more than 4 months or multiple shorter events.
PTF observations detected the outburst in individual images, suggesting that the outburst was already active 11 months prior to the explosion. All of the PTF photometric measurements are listed in Table 2. We note that PTF did not observe the SN itself. Assuming there was a single precursor, the properties and energetics of this event are listed in Table 4.

\subsection{PTF 10bjb}

PTF 10bjb was discovered by PTF on 2010 February 16, and the only spectrum obtained on 2010 March 7 resembles those of luminous blue variables and SNe IIn. Close inspection of the light curve suggests that the SN was discovered while in a pre-explosion high state, and that the spectrum was obtained prior to the explosion. The spectrum shows Balmer emission lines, the broader component of which has a velocity $(\sigma)$ of about $600 \mathrm{~km} \mathrm{~s}^{-1}$. Also detected are emission lines of He I and $\mathrm{Ca}$ II. The He I $\lambda 5876$ line exhibits a narrow P-Cygni profile with a velocity of $\sim 600 \mathrm{~km} \mathrm{~s}^{-1}$, while the $\mathrm{H} \alpha$ line has a P-Cygni profile with a velocity of $\sim 300 \mathrm{~km} \mathrm{~s}^{-1}$. The spectrum of this event is presented in Figure 6.

The light curve of PTF 10bjb is shown in Figure 7. Between $\sim 80$ days up to 0 days prior to the assumed explosion time of this SN, which is based on the fast rise in the SN light curve, we detect a significant excess in the flux residuals. This excess had a peak absolute magnitude of -15.1 and it lasted for $\sim 110$ days. Two years after $t_{\text {rise }}$, the $\mathrm{SN}$ is detected at a flux level which is a factor of $\sim 5$ dimmer than the pre-explosion outburst. Assuming zero bolometric correction, the peak bolometric luminosity of this outburst is $\sim 3 \times 10^{41} \mathrm{erg} \mathrm{s}^{-1}$, and its radiated energy is $\sim 2.6 \times 10^{48} \mathrm{erg}$.

In addition, there is a possible detection of the progenitor at an absolute magnitude of roughly -14 about a year prior to the $\mathrm{SN}$ explosion. However, given that there is a single detection at this time, we do not consider it to be a good precursor candidate (see Section 4.2). The physical parameters of the SN and the precursor are listed in Table 4.

\subsection{SN $2011 \mathrm{hw}$}

SN 2011hw was discovered by Dintinjana et al. (2011) on 2011 November 18.7, at apparent magnitude 15.7. Valenti et al. (2011) reported that a spectrum obtained on 2011 November 


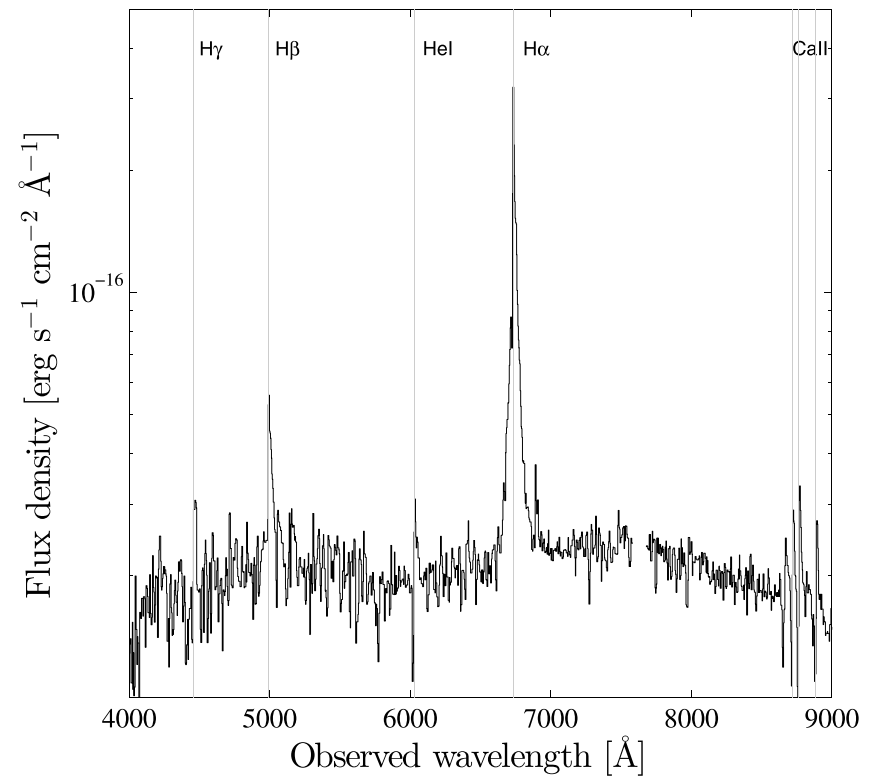

Figure 6. Spectrum of PTF 10bjb as obtained by Keck-I/LRIS on 2010 March 7.

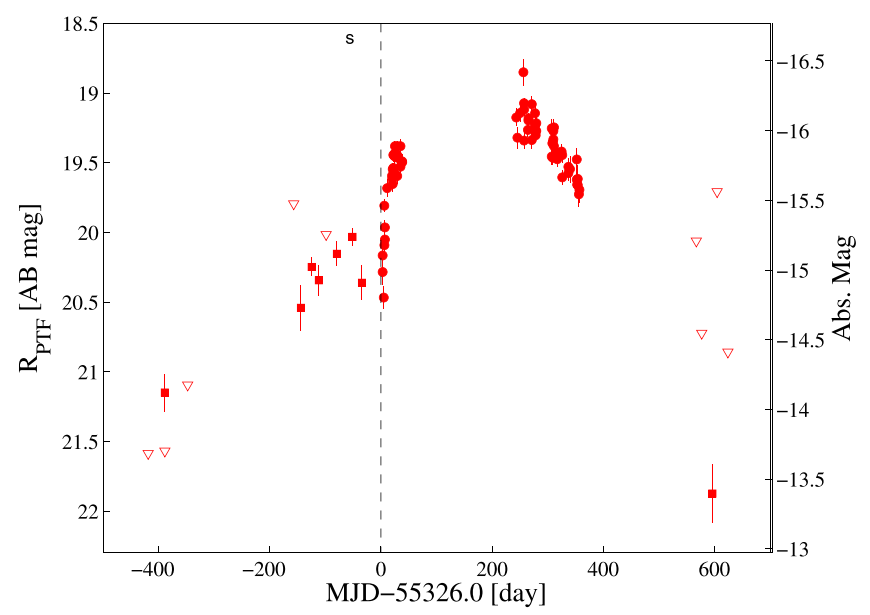

Figure 7. Light curve of PTF 10bjb exhibits a clear outburst lasting for $\sim 4$ months prior to the explosion (identified by the fast rise in the light curve). An additional possible detection is seen $\sim 400$ days prior to the explosion, but we do not consider it to be a good candidate since it is based on a single binned detection (see the discussion about false-alarm probability in Section 4.2). The upper limits corresponds to $5 \sigma$ bounds calculated in 15 day bins.

(A color version of this figure is available in the online journal.)

19.8 shows it to be similar to the transitional Type IIn/Ibn SN 2005la (Pastorello et al. 2008). The spectrum is blue and exhibits emission lines of $\mathrm{H}$ and $\mathrm{He}$. The most prominent $\mathrm{He}$ I lines compete in strength with $\mathrm{H} \alpha$. The FWHM velocity of $\mathrm{H} \alpha$ is $2700 \mathrm{~km} \mathrm{~s}^{-1}$, while that of He I $\lambda 5876$ is $\sim 2000 \mathrm{~km} \mathrm{~s}^{-1}$. We note that the apparent magnitude of this SN reported by Dintinjana \& Mikuz (2011) corresponds to an absolute magnitude of about -19.3 . Our initial search suggested that there is a detection of an outburst $\sim 3$ months prior to the SN explosion. However, since this is based on a single detection, we do not consider it to be a good candidate.

\subsection{PTF 10weh}

PTF 10weh was discovered by PTF on 2010 September 22.14. The SN brightened to a peak absolute magnitude of about -20.7

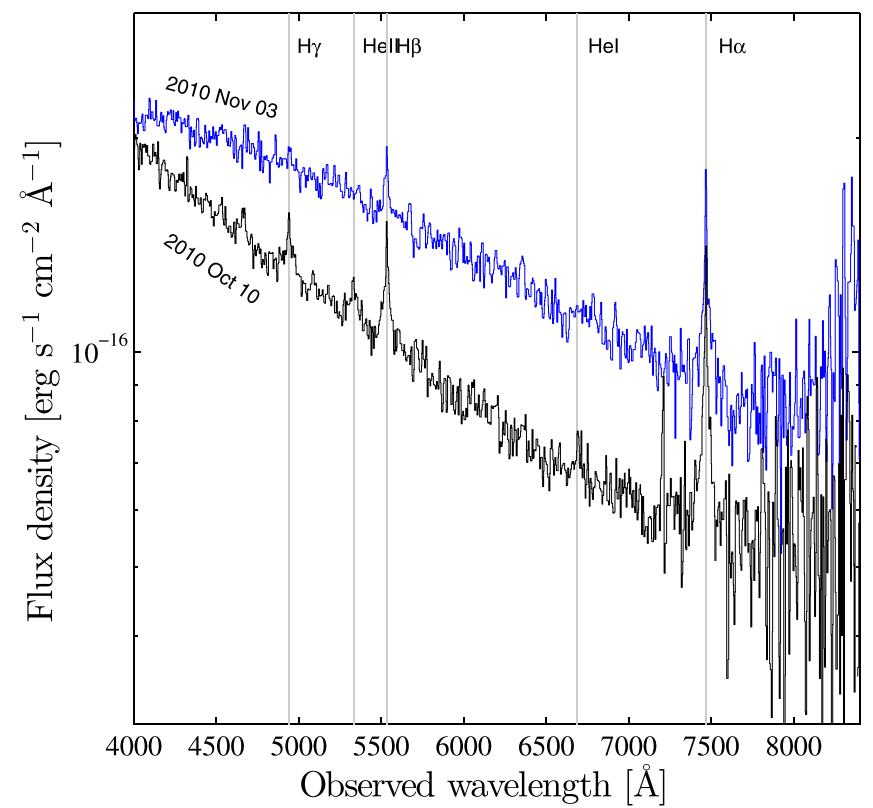

Figure 8. Spectra of PTF 10weh as obtained using the KPNO $4 \mathrm{~m}$ telescope equipped with the RC spectrograph.

(A color version of this figure is available in the online journal.)

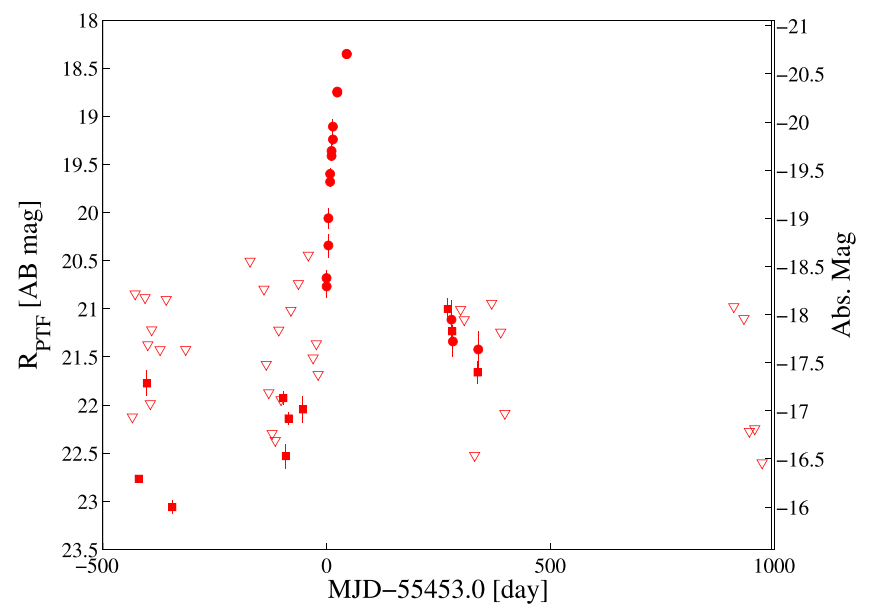

Figure 9. PTF $R$-band light curve of PTF 10weh. Filled circles show individual measurements, while filled boxes mark three-day binned measurements with at least two measurements per bin. The empty triangles denote $5 \sigma$ upper limits. The bin size is 3 days prior to the SN explosion and 15 days after the SN explosion.

(A color version of this figure is available in the online journal.)

over $\sim 40$ days. A spectrum taken on 2010 October 10 exhibits a blue continuum together with Balmer emission lines as well as $\mathrm{He}$ I and He II. The broadest component of the $\mathrm{H} \alpha$ line has a velocity $(\sigma)$ of $\sim 1000 \mathrm{~km} \mathrm{~s}^{-1}$. The first two spectra of this source are shown in Figure 8.

The light curve of this $\mathrm{SN}$ and the candidate precursor event is shown in Figure 9. There is a possible precursor $\sim 3$ months prior to the $\mathrm{SN}$ rise, lasting for $\sim 40$ days. A close-up view of the precursor light curve is presented in Figure 10. Figure 11 gives another version of the precursor light curve, showing all of its individual flux measurements, along with flux measurements at random positions in the host galaxy and other nearby galaxies. Inspection of the data reveals that the detection depends on the binning scheme. However, 


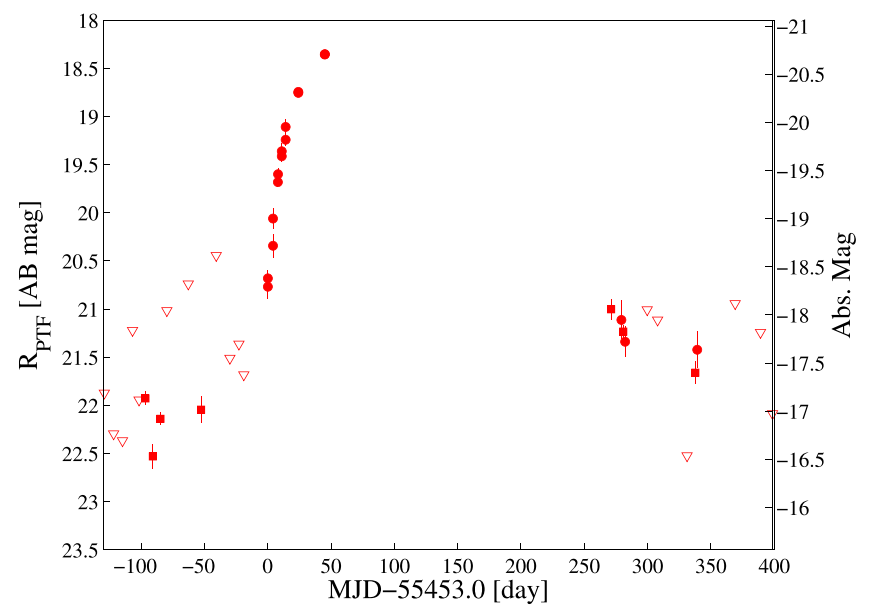

Figure 10. Close-up view of the PTF $R$-band light curve of PTF 10weh around the precursor candidate time (see also Figure 9). Filled circles denote individual measurements, while filled boxes mark 3 day binned measurements with at least two measurements per bin. The empty triangles are $5 \sigma$ upper limits. The bin size is 3 days prior to the $\mathrm{SN}$ explosion and 15 days after the $\mathrm{SN}$ explosion.

(A color version of this figure is available in the online journal.)

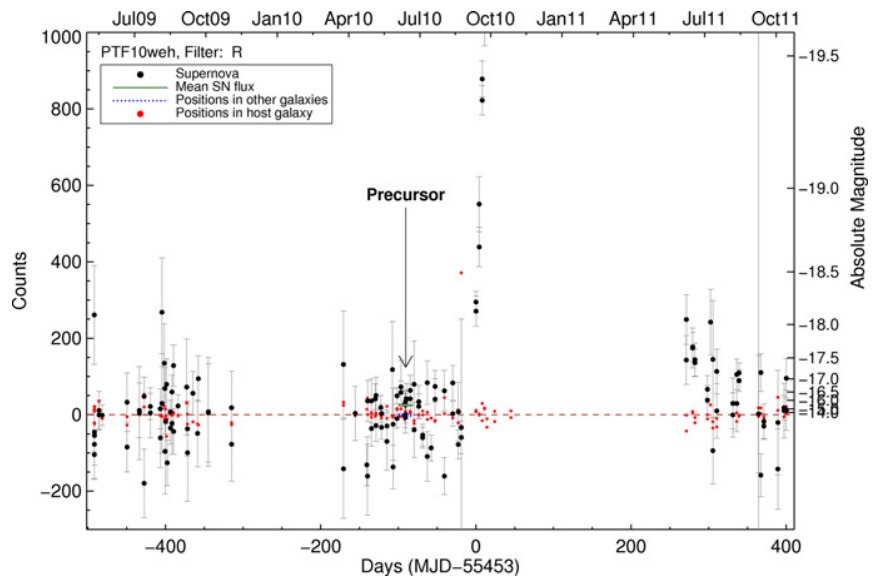

Figure 11. Counts-space light curve of SN PTF 10weh. Each black circle represents a point-spread function (PSF) flux measurement on an individual PTF exposure after image subtraction. The precursor is labeled, and the mean precursor flux is shown as a green horizontal line, with the hashed area representing the uncertainty. The blue line indicates the average of random measurements in other galaxies in the image, illustrating the typical flux uncertainty and residual in the flux measurements after image subtraction. The red circles show the average of random measurements in the same host as the $\mathrm{SN}$ (where the host is extended enough to allow this test), tracking the random subtraction noise that may affect the flux measurements. The horizontal red line denotes the mean of the red circles, and is consistent with zero flux as expected after image subtraction. The absolute magnitude scale is shown on the righthand ordinate for reference, and the abscissa shows the time in days relative to the $\mathrm{SN} t_{\text {rise. }}$. This figure is shown here mainly to demonstrate the various noise levels.

(A color version of this figure is available in the online journal.)

given that there are four temporally adjacent points that deviate by more than $5 \sigma$, we consider this to be a good precursor candidate. The absolute magnitude of this precursor is about -17 . Assuming zero bolometric correction, this corresponds to a luminosity of $\sim 1.9 \times 10^{42} \mathrm{erg} \mathrm{s}^{-1}$. The lower limit on the radiated energy of the precursor is $\sim 5 \times 10^{48} \mathrm{erg}$. The physical parameters of the SN and its outburst are listed in Table 4.

The pre-explosion light curve of this $\mathrm{SN}$ also reveals additional possible detections 11-14 months prior to the SN explo-

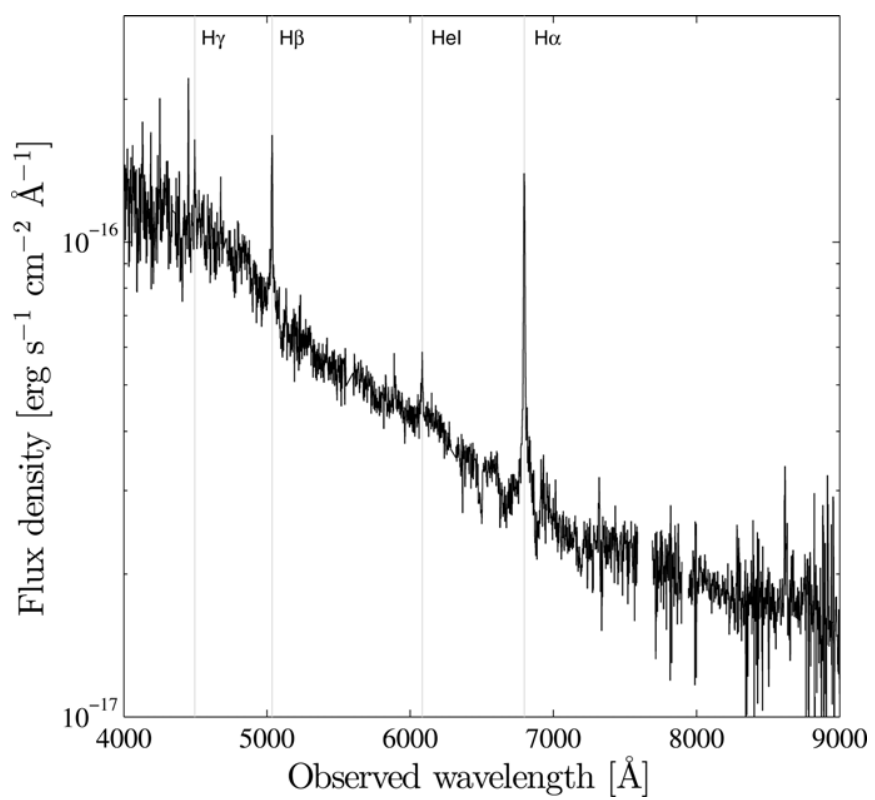

Figure 12. Spectrum of PTF 12cxj as obtained with the Gemini-N telescope equipped with the GMOS spectrograph.

sion. However, given that these detections are not consecutive in time, we do not regard them as good precursor candidates.

\subsection{PTF $12 c x j$}

PTF 12cxj was discovered by PTF on 2012 April 16.14. The $\mathrm{SN}$ rose to an $R$-band absolute magnitude of -17.2 over two weeks. The spectrum of the SN, presented in Figure 12, was obtained on 2012 April 18 during the early rise of the SN. The spectrum has Balmer and He I lines in emission. Fitting a two-Gaussian model to the $\mathrm{H} \alpha$ line shows that the broadest component has a velocity width $(\sigma)$ of $1200 \mathrm{~km} \mathrm{~s}^{-1}$.

This SN has a large number of pre-explosion observations, and it shows a single detection $\sim 384$ days prior to its $t_{\text {rise }}$. Closer inspection reveals that this detection depends on the binning scheme, and that with shorter bins of four days, multiple detections at an absolute magnitude of about -13.5 are seen. Moreover, inspection of the $g$-band data reveals a marginal detection around -435 days prior to $t_{\text {rise }}$. The full $R$-band light curve is illustrated in Figure 13. The most convincing precursor candidate events are detected about two weeks and 700 days prior to $t_{\text {rise }}$. Given the large number of observations of this SN field, the light curve in this plot utilized two-day bins and $4 \sigma$ upper limits. A close-up view of the light curve of the two precursors is shown in Figure 14. The $R$-band peak absolute magnitudes of these candidate precursors are -14.8 and -13 . However, the candidate precursor two weeks prior to the explosion is very close in time to the $\mathrm{SN}$ fast rise and therefore may be regarded as part of the $\mathrm{SN}$ rise. As seen in Figure 14, the two detections are followed by a nondetection which is about a magnitude deeper than the possible detection at -8 days prior to $t_{\text {rise }}$. Furthermore, a $t^{2}$ extrapolation to the $\mathrm{SN}$ rise flux suggests that the $\mathrm{SN}$ started its rise after this candidate precursor. We cannot rule out the possibility that this precursor is part of the SN light curve (e.g., similar to SN 2011dh; Arcavi et al. 2011).

As before, Figure 15 shows another version of the precursor light curve, with all of the individual flux measurements, along with flux measurements in random positions on nearby galaxies and the same host galaxy. 


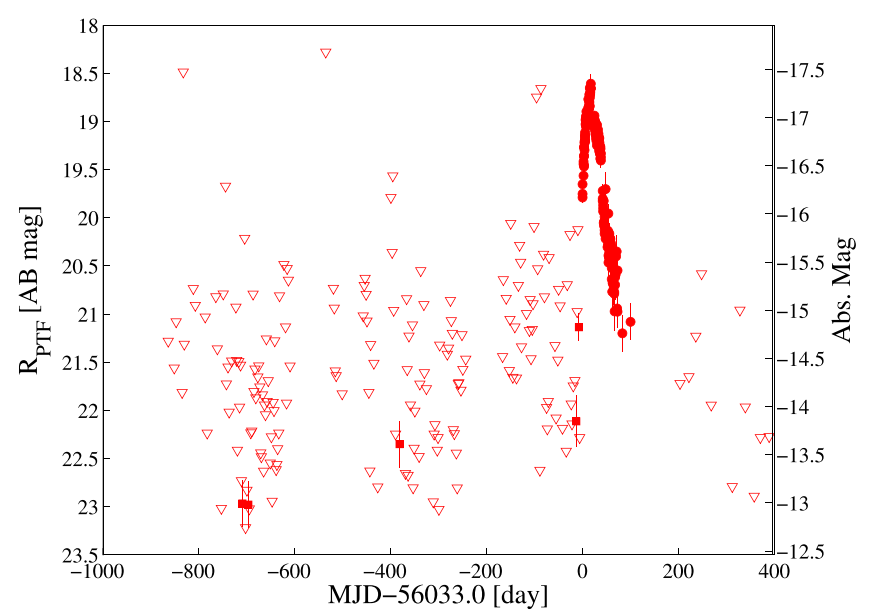

Figure 13. PTF $R$-band light curve of PTF 12cxj. Filled circles denote individual measurements, while filled boxes mark binned measurements (all the filled symbols prior to time 0 are filled boxes). The empty triangles show $4 \sigma$ upper limits. The bin size is two days prior to the $\mathrm{SN}$ explosion. The minimum number of measurements in each bin is 4 .

(A color version of this figure is available in the online journal.)

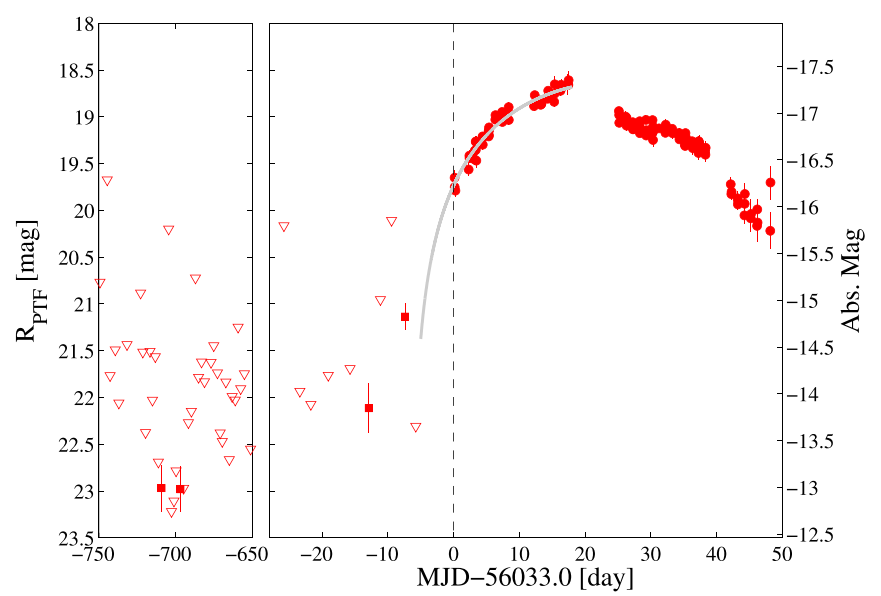

Figure 14. Light curve of PTF 12cxj shows two possible precursors (based on multiple consecutive detections, where single detections are ignored). The upper limits corresponds to $4 \sigma$ bounds calculated in two-day bins. Extrapolation of the light curve, based on a $t^{2}$ law (gray line), suggests that the detections visible at around -10 days took place before the SN explosion (see also Figure 4). Furthermore, there is a deep nondetection between these detections and the assumed time of the SN explosion. We note that even if we remove this event from our sample, the rate of precursors does not change by much, and the correlations (Figure 19) are still detected.

(A color version of this figure is available in the online journal.)

The second candidate precursor is detected $\sim 700$ days prior to the SN explosion, and it is also based on two marginal detections separated by about two weeks. If real, the peak absolute $R$-band magnitude of this precursor is about -13 .

For all of the $\mathrm{SNe}$ in our sample, we also calculated the autocorrelation function of the flux residuals prior to $t_{\text {rise }}$. The only $\mathrm{SNe}$ which exhibited significant (at the $3 \sigma$ level) autocorrelation at lag one (i.e., corresponding to two successive measurements) are PTF $12 \mathrm{cxj}(4.7 \sigma)$ and PTF 10tel $(3.2 \sigma)$. Figure 16 presents the discrete autocorrelation function (Edelson \& Krolik 1988) of all the flux residuals of PTF 12cxj, measured before $t_{\text {rise }}$. The figure shows significant autocorrelation on timescales of a few days to ten days. This may indicate that the flux residuals are not pure uncorrelated noise, but contain

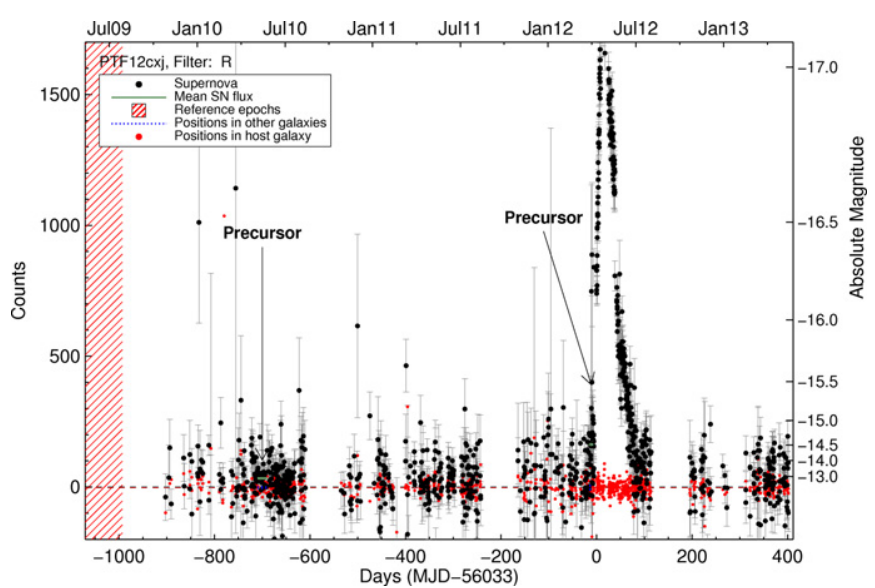

Figure 15. As in Figure 11, but for PTF 12cxj. This figure is shown here mainly to demonstrate the various noise levels. A clearer picture of the precursors is shown in Figures 13-14.

(A color version of this figure is available in the online journal.)

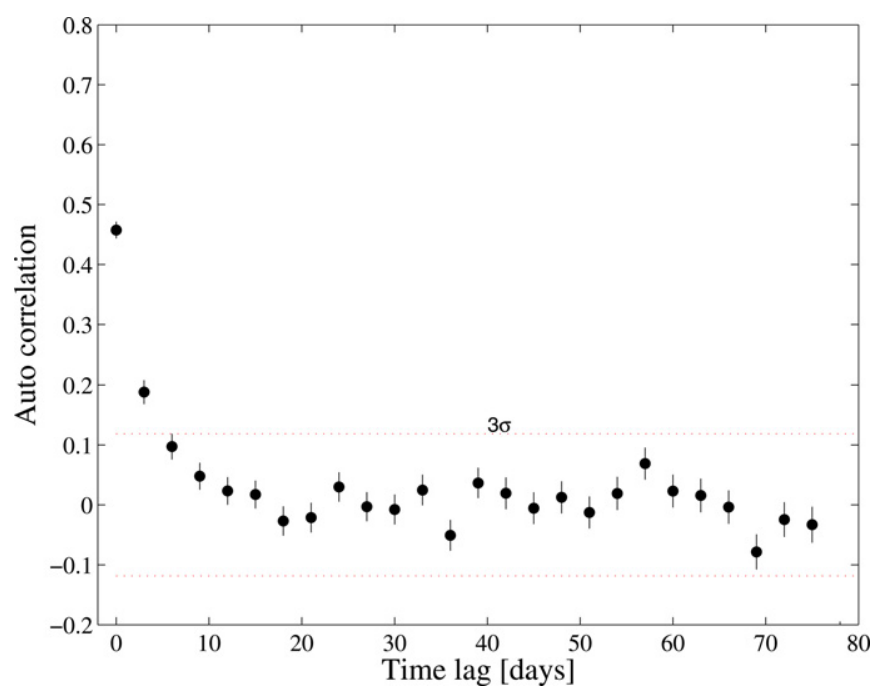

Figure 16. Discrete autocorrelation function (Edelson \& Krolik 1988) of the flux residuals of PTF $12 \mathrm{cxj}$, taken before $t_{\text {rise }}$. In order to calculate the autocorrelation function we use time bins of three days. The dotted horizontal lines represent the lower and upper $3 \sigma$ bounds, estimated assuming $\sigma \approx 1 / \sqrt{N}$, where $N$ is the number of measurements in each time bin.

(A color version of this figure is available in the online journal.)

a fraction of the progenitor light. Moreover, it is possible that the progenitor is variable on timescales of a few days. An alternative explanation to the variability is that this signal is caused by the lunar synodic period (i.e., the limiting magnitude is better during dark time). Nevertheless, this means that the progenitor of PTF 12cxj is likely detected in several binned images, and that its absolute $R$-band magnitude is about -13 , brighter even than the possible progenitor of SN 2010jl/PTF 10aaxf (Smith et al. 2011). Given the detection of a signal in the autocorrelation, we consider the two precursor events as real, and their properties are listed in Table 4.

\section{CONTROL TIME}

In order to calculate the rate of $\mathrm{SN}$ precursors, we need to estimate the "control time"- - that is, for how long each SN location was observed (prior to its explosion) to a given limiting magnitude. Table 5 lists, for each SN, the time bin windows (of 15 days) prior to the $\mathrm{SN}$ explosion and the $5 \sigma$ sensitivity depth at 


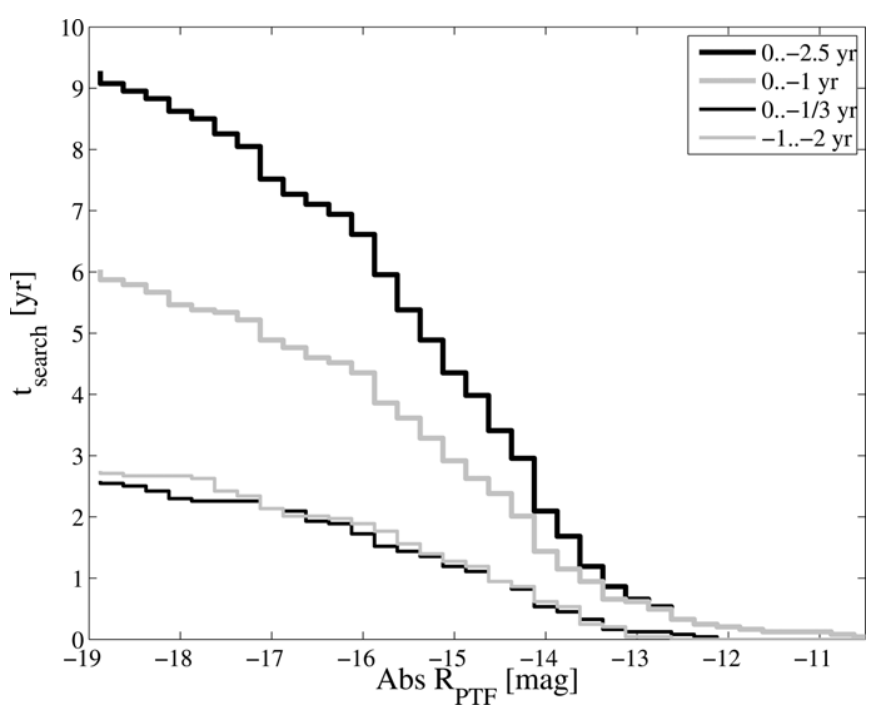

Figure 17. Cumulative precursor search control time in years as a function of absolute magnitude. For each time range prior to the SN explosion (see legend), this plot shows the total number of years we searched our entire sample for precursors down to a given limiting magnitude (see text for details).

Table 5

Control Time

\begin{tabular}{lcccc}
\hline \hline Name & $\begin{array}{c}\Delta t \\
(\text { day })\end{array}$ & $\begin{array}{c}R_{\text {PTF }} \\
(\mathrm{mag})\end{array}$ & $\begin{array}{c}\text { Abs. } R_{\text {PTF }} \\
(\mathrm{mag})\end{array}$ & $N_{\text {meas }}$ \\
\hline PTF10aaxf & -228.6 & 21.4 & -12.0 & 6 \\
PTF10aaxf & -203.8 & 20.9 & -12.6 & 1 \\
PTF10aaxf & -192.3 & 20.0 & -13.4 & 1 \\
PTF10aaxf & -179.8 & 20.7 & -12.7 & 1 \\
PTF10aazn & -306.6 & 21.5 & -12.8 & 8 \\
\hline
\end{tabular}

Notes. Precursor search limiting magnitude (apparent and absolute) in time windows. $\Delta t$ is the mean time of the measurements within each 15 day bin as measured relative to $t_{\text {rise }}$ of the SN (see Table 1). $N_{\text {meas }}$ is the number of data points in each 15 day bin. For bins with $N_{\text {meas }}<6$ we present the median of all $6 \sigma$ limiting magnitudes in the bin. We mark instances with $N_{\text {meas }}=1$ (even if $N_{\text {meas }}>1$ ).

(This table is available in its entirety in a machine-readable form in the online journal. A portion is shown here for guidance regarding its form and content.)

each window for bins with more than five measurements (second channel), or the median $6 \sigma$ limiting magnitudes at windows with fewer than six measurements (first channel).

To calculate the control time as a function of absolute magnitude, we sum over all the SNe the number of time bins (in Table 5) in which the limiting absolute magnitude is deeper than the absolute magnitude of interest, and multiply by the bin size (i.e., 15 days). Figure 17 displays the sample cumulative control time as a function of absolute $R$-band magnitude. Specifically, this plot shows for several time ranges (e.g., 0-2.5 yr prior to the SN explosion; black line) the total number of years, within a time range, in which we are able to detect a precursor event brighter than a given absolute magnitude.

Figure 18 shows the sample cumulative control time fraction as a function of absolute $R$-band magnitude. This is defined as the total amount of time (given in Figure 17) divided by the size of the time window (listed in the legend). This fraction represents the equivalent number of $\mathrm{SNe}$ observed during the entire time window as a function of absolute limiting magnitude. The plot demonstrates that the highest efficiency is available for

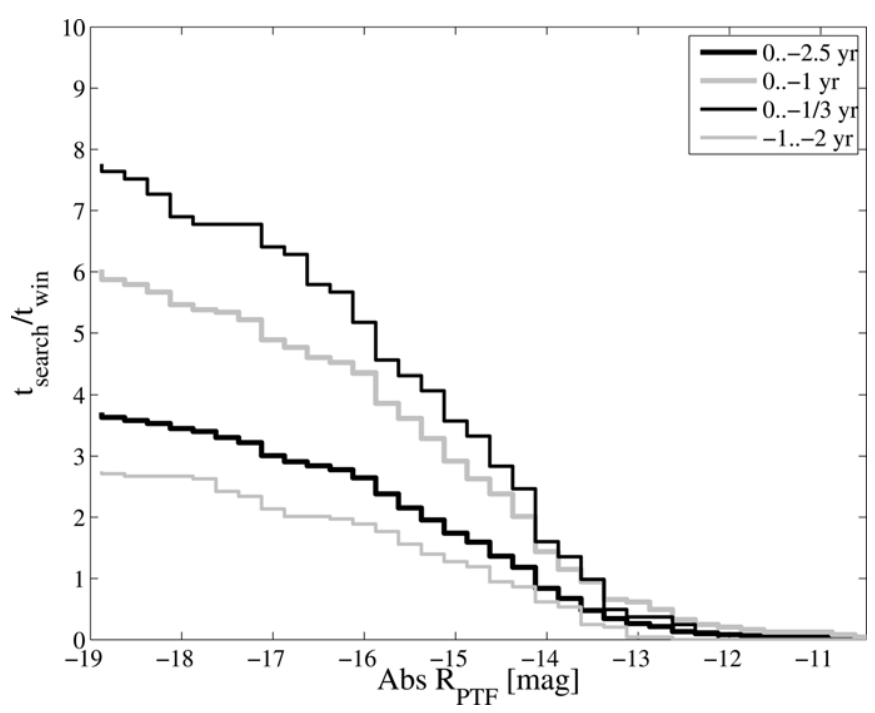

Figure 18. Cumulative precursor search control time fraction as a function of absolute magnitude (Equation (1)). For each time range prior to the SN explosion (see legend), this plot shows the total number of SNe we observed for the equivalent of the entire search window down to a given limiting magnitude.

relatively short periods prior to the $\mathrm{SN}$ explosion. This is due to the fact that PTF switches many of the observed fields typically every 3-4 months, thereby probably explaining why many of the precursors we find are within 100 days before the SN explosion.

\section{PRECURSOR RATE}

Next, we would like to estimate the rate of the precursors. Such a rate estimate should be regarded as an order-ofmagnitude estimate, mostly because it is affected by large Poisson errors and complicated biases.

Given the cumulative control time $t_{\text {search }}\left(<M_{R}\right)$ over all SNe as a function of absolute magnitude $\left(M_{R}\right)$, and the cumulative control time fraction

$$
f_{\text {search }}\left(<M_{R}\right)=\frac{t_{\text {search }}\left(<M_{R}\right)}{t_{\text {win }}},
$$

where $t_{\text {win }}$ is the length of the search window considered (e.g., $1 / 3 \mathrm{yr}$ prior to the explosion), we can calculate the average (over all $\mathrm{SNe}$ ) rate of precursors. The mean precursor rate per $\mathrm{SN}$, in units of events per unit time, is

$$
R_{\text {prec }}=\frac{N_{\text {prec }}}{\left(t_{\text {search }}\left[<M_{R}\right]\right)},
$$

where $N_{\text {prec }}$ is the total number of precursor events detected in the sample within $t_{\text {win }}$ (this can be more than one precursor per $\mathrm{SN}$ ). The fraction of SNe IIn that show at least one precursor event within $t_{\text {win }}$ is given by

$$
f_{\text {prec }}=\frac{N_{\mathrm{SN}, \text { prec }}}{\left(f_{\text {search }}\left[<M_{R}\right]\right)} .
$$

Here, $N_{\mathrm{SN} \text {,prec }}$ is the number of $\mathrm{SNe}$ that exhibit at least one precursor event within $t_{\text {win }}$ (i.e., each $\mathrm{SN}$ with detected precursors is counted once regardless of the number of precursor events).

Remarkably, we find that the fraction of SNe IIn that have precursors is of order unity. Assuming a homogeneous population, at the one-sided $99 \%$ confidence level, more than $52 \%(98 \%)$ 
Table 6

Rate of Precursors

\begin{tabular}{|c|c|c|c|c|c|c|c|}
\hline$N_{\mathrm{SN}, \text { prec }}$ & $N_{\text {prec }}$ & $\begin{array}{c}M_{R} \\
\text { (mag) }\end{array}$ & $\begin{array}{l}t_{\text {win }} \\
(\mathrm{yr})\end{array}$ & $\begin{array}{c}t_{\text {search }}\left(<M_{R}\right) \\
(\mathrm{yr})\end{array}$ & $f_{\text {search }}\left(<M_{R}\right)$ & $\begin{array}{c}\mathcal{R}_{\text {prec }}\left(<M_{R}\right) \\
\left(\mathrm{yr}^{-1}\right)\end{array}$ & $f_{\text {prec }}\left(<M_{R}\right)$ \\
\hline 4 & 4 & -14.0 & $0.00 . .-0.33$ & 0.53 & 1.60 & $\begin{array}{c}7.49_{-3.59,-5.51}^{+5.92,+11.96} \\
\end{array}$ & $2.50_{-1.20,-1.84}^{+1.97,+3.99}$ \\
\hline 4 & 4 & -14.0 & $0.00 . .-1.00$ & 1.44 & 1.44 & $2.78_{-1.33,-2.05}^{+2.20,+4.44}$ & $2.78_{-1.33,-2.05}^{+2.20,+4.44}$ \\
\hline 4 & 4 & -14.0 & $0.00 . .-2.50$ & 2.09 & 0.84 & $1.91_{-0.91,-1.40}^{+1.51,+3.05}$ & $4.77_{-2.29,-3.51}^{+3.78,+7.62}$ \\
\hline 5 & 5 & -11.8 & $0.00 . .-1.00$ & 0.16 & 0.16 & $30.44_{-13.15,-20.80}^{+20.59,+41.52}$ & $30.44_{-13.15,-20.80}^{+20.59,+41.52}$ \\
\hline 5 & 6 & -11.8 & $0.00 . .-2.50$ & 0.16 & 0.07 & $36.52_{-14.49,-23.42}^{+21.82,+43.95}$ & $\begin{array}{r}76.09_{-32.87,-52.00}^{+51.48,+103.80} \\
\end{array}$ \\
\hline 2 & 2 & -14.0 & $0.00 . .-0.33$ & 0.53 & 1.60 & $3.75_{-2.42,-3.32}^{+4.94,+10.02}$ & $1.25_{-0.81,-1.16}^{+1.65,+4.00}$ \\
\hline 2 & 2 & -14.0 & $0.00 . .-1.00$ & 1.44 & 1.44 & $1.39_{-0.90,-1.23}^{+1.84,+3.72}$ & $1.39_{-0.90,-1.29}^{+1.84,+4.46}$ \\
\hline
\end{tabular}

Notes. Average fraction of SNe IIn with precursors $\left(f_{\text {prec }}\left[<M_{R}\right]\right)$ and rate of precursors $\left(\mathcal{R}_{\text {prec }}\left[<M_{R}\right]\right)$ based on SNe in our sample. The fractions and rates are listed for various time windows prior to the $\mathrm{SN}$ explosion and different absolute limiting magnitude $\left(M_{R}\right)$. The $2 \sigma$ and $3 \sigma$ limit refers to two-sided probabilities (Gehrels 1986). The number of events listed below the horizontal line assumes that the precursors detected in binned data (i.e., from PTF 10weh and PTF 12cxj) are not real.

of SNe IIn have at least one pre-explosion outburst brighter than an absolute magnitude of -14 , and that takes place up to $1 / 3$ (2.5) yr prior to the $\mathrm{SN}$ explosion. Furthermore, our results suggest that, typically, SNe IIn exhibit more than one precursor on a timescale of 1 yr prior to the explosion. Specifically, during this final year prior to the explosion, the average rate of precursors brighter than absolute magnitude -14 , is $\mathcal{R}=7.5_{-3.6,-5.5}^{+5.9,+12.0} \mathrm{yr}^{-1}$ ( $1 \sigma$ and $2 \sigma$ uncertainties $)$. We note that this estimate contains only Poisson errors, and does not include the prior that the number of events cannot exceed one year divided by the typical duration of each event. Furthermore, even if we assume that the precursors of PTF 10weh and PTF 12cxj are not real (i.e., excluding events detected only in coadded images), the derived rate is $\mathcal{R}=3.8_{-2.4,-3.3}^{+4.9} \mathrm{yr}^{-1}$, still likely above unity. We note that besides the Poisson errors taken into account in our rate estimate, there are other factors which are more complicated to model. These include systematic biases, the purity of our sample, the duration of the precursors, and whether multiple rebrightening of the same source should be counted as a single event. However, at this stage we claim that the largest source of error is the Poisson error (due to the small number of events).

Our analysis also suggests that fainter precursors are even more common, and that the cumulative luminosity function of precursors is roughly $\mathcal{R}\left(>L_{\text {peak }}\right) \propto L_{\text {peak }}^{-0.7 \pm 0.5}$, where $\mathcal{R}\left(>L_{\text {peak }}\right)$ is the rate of precursors brighter than peak luminosity $L_{\text {peak }}$. We note that these statistical results are in accord with individual well-studied cases like SN 2009ip, in which several precursors were documented (e.g., Prieto et al. 2013; Margutti et al. 2014).

The derived precursor rate and fraction of SNe with precursors, along with their $1 \sigma$ and $2 \sigma$ uncertainties (Gehrels 1986), are listed in Table 6; it suggests that the rate of precursors is larger than unity. This may indicate that on average, each SN has more than one precursor event in the few years prior to their explosion, as in SN 2009ip.

A disadvantage of using the combined control time of all the $\mathrm{SNe}$ is that it assumes the precursor properties are universal among all SNe IIn. Another caveat is that our search is mostly sensitive to events with a duration longer than about two weeks. Shorter events can be detected but their control time suffers from additional uncertainties. In any case, our approach still gives us an estimate for the rate of precursors.

\section{CSM MASS ESTIMATE}

A possible scenario is that the precursors are associated with mass-loss events. Two scenarios can then be considered. In the first scenario, part of the CSM kinetic energy is converted into radiated energy. We therefore assume that up to an efficiency factor $\epsilon$, the kinetic energy of the CSM is equivalent to its radiated energy. In this case,

$$
M_{\mathrm{CSM}} \approx \epsilon \frac{2 L_{\mathrm{prec}} \delta t}{v^{2}}
$$

Here $L_{\text {prec }}$ is the mean luminosity of the outburst, $\delta t$ is its duration, and $v$ is the velocity of the CSM. We estimated the CSM velocity based on the width of the broad component of the $\mathrm{H} \alpha$ emission line detected in the SN spectra (Table 4).

In an alternative scenario, the CSM and precursor can still be related, but in an opposite way. Instead of transferring energy from the CSM to the precursor, the CSM could be the result of the precursor luminosity. This is naturally obtained if the CSM was accelerated through a continuum-driven wind (e.g., Shaviv 2000; Smith \& Owocki 2006), such that

$$
M_{\mathrm{CSM}, \mathrm{cont}} \approx W \frac{L_{\mathrm{prec}} \delta t}{c_{\mathrm{s}} c}
$$

Here $c_{\mathrm{S}}$ is the speed of sound at the base of the optically thick wind $\left(\sim 60 \mathrm{~km} \mathrm{~s}^{-1}\right), c$ is the speed of light, and $W \approx 5$ is an empirically derived constant (Shaviv 2000, 2001). Although the flow of energy in the two scenarios is opposite, we note that the second scenario is equivalent to the first with a universal velocity and it does not depend on the CSM velocity via the measured line widths.

\section{CORRELATION BETWEEN THE PRECURSOR AND SN PROPERTIES}

The properties of the candidate precursors and their corresponding SN light curves are presented in Table 4. We also added to this table SN 2009ip, for which similar outbursts were detected and characterized. If these SN light curves are powered by shock breakout followed by interaction of the SN ejecta with a dense CSM, then we expect that some of the outburst and SN properties will be correlated (Ofek et al. 2010, Ofek et al. 2014a, 2014b; Svirski et al. 2012). If a substantial fraction of 

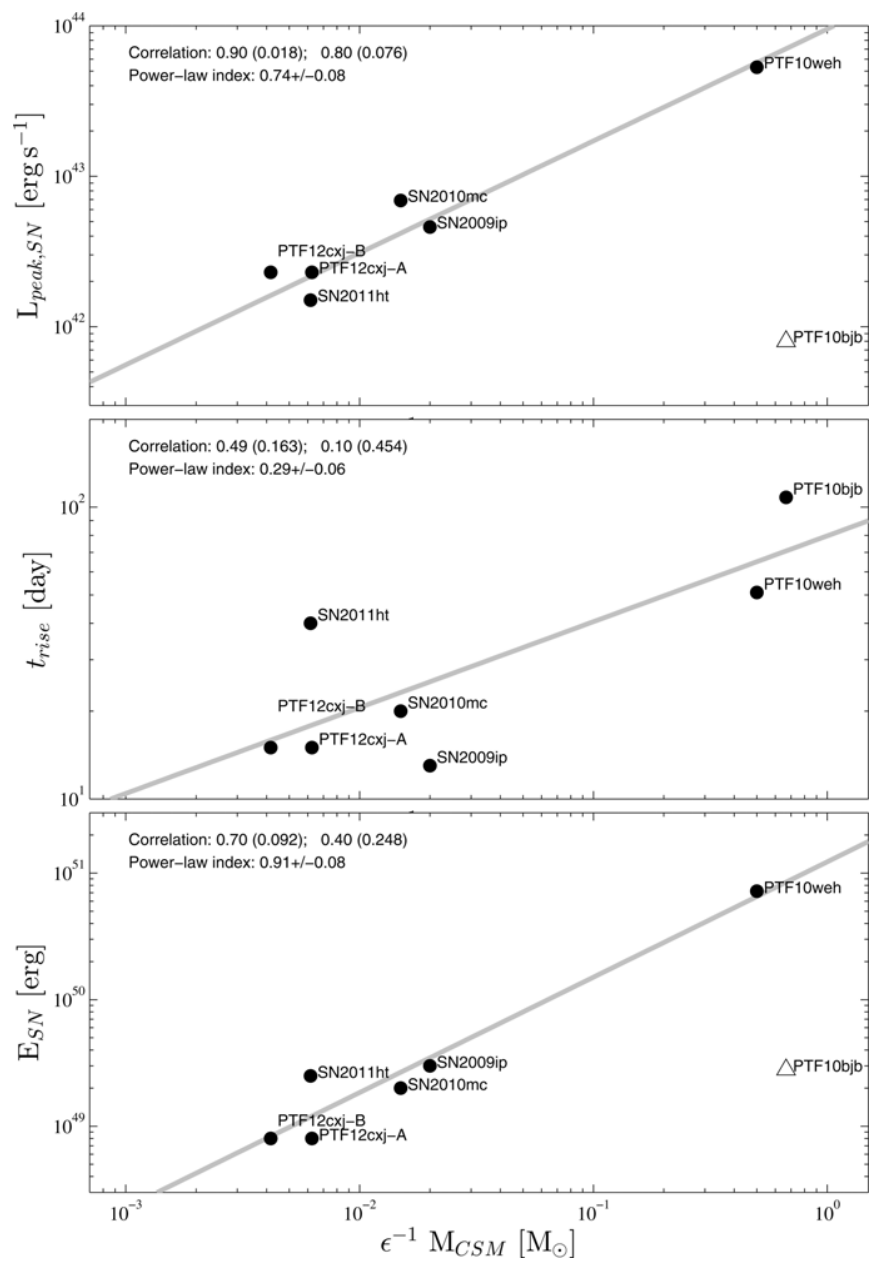

Figure 19. Correlations between the SN properties and the ejected CSM mass estimated using Equation (4). Top: SN $R$-band peak luminosity vs. CSM mass. Middle: $\mathrm{SN}$ rise time vs. CSM mass. Bottom: $\mathrm{SN}$ integrated $R$-band luminosity vs. CSM mass. At the top of each panel, we also provide the Spearman rank correlation coefficient and the probability of getting this correlation by chance. This false-alarm probability was calculated using the bootstrap technique (Efron 1982). Two correlations are given: the first is for the entire sample, while the second excludes PTF 10weh. The best-fit power law is shown with a gray line, and the power-law index is provided below the correlation coefficient. The correlation and power law were calculated excluding the PTF $12 \mathrm{cxj}-\mathrm{B}$ event. Excluding the PTF $12 \mathrm{cxj}-\mathrm{A}$ event or combining the two points of PTF $12 \mathrm{cxj}$ into one (i.e., by adding the estimated CSM mass) have a small impact on the results. We note that the relative errors on each data point are estimated to be on the order of tens percent. However, the use of the bootstrap technique gives us a robust estimate for the false-alarm probability, regardless of the poorly known errors. Furthermore, if we exclude PTF 12cxj and PTF 10weh (detected via the second channel), then the sample is too small to detect any correlations. Therefore, a larger sample is required in order to verify these correlations.

the $\mathrm{SN}$ radiated energy originates from the interaction of the SN ejecta with the CSM, then this material mass will determine the total (and peak) radiated luminosity of the SN. Moreover, the CSM mass will determine the diffusion timescale and therefore the $\mathrm{SN}$ rise time. Given this prediction, we search for such correlations.

To estimate the CSM mass, we used Equation (4) or Equation (5). We note that this approach assumes that $\epsilon$ is on the same order of magnitude for all the precursors in our sample. The three panels in Figure 19 show (from top to bottom) the precursor-ejected CSM mass based on Equation (4), as a function of the SN $R$-band peak luminosity, rise time, and integrated $R$-band energy. At the top of each panel, we also provide
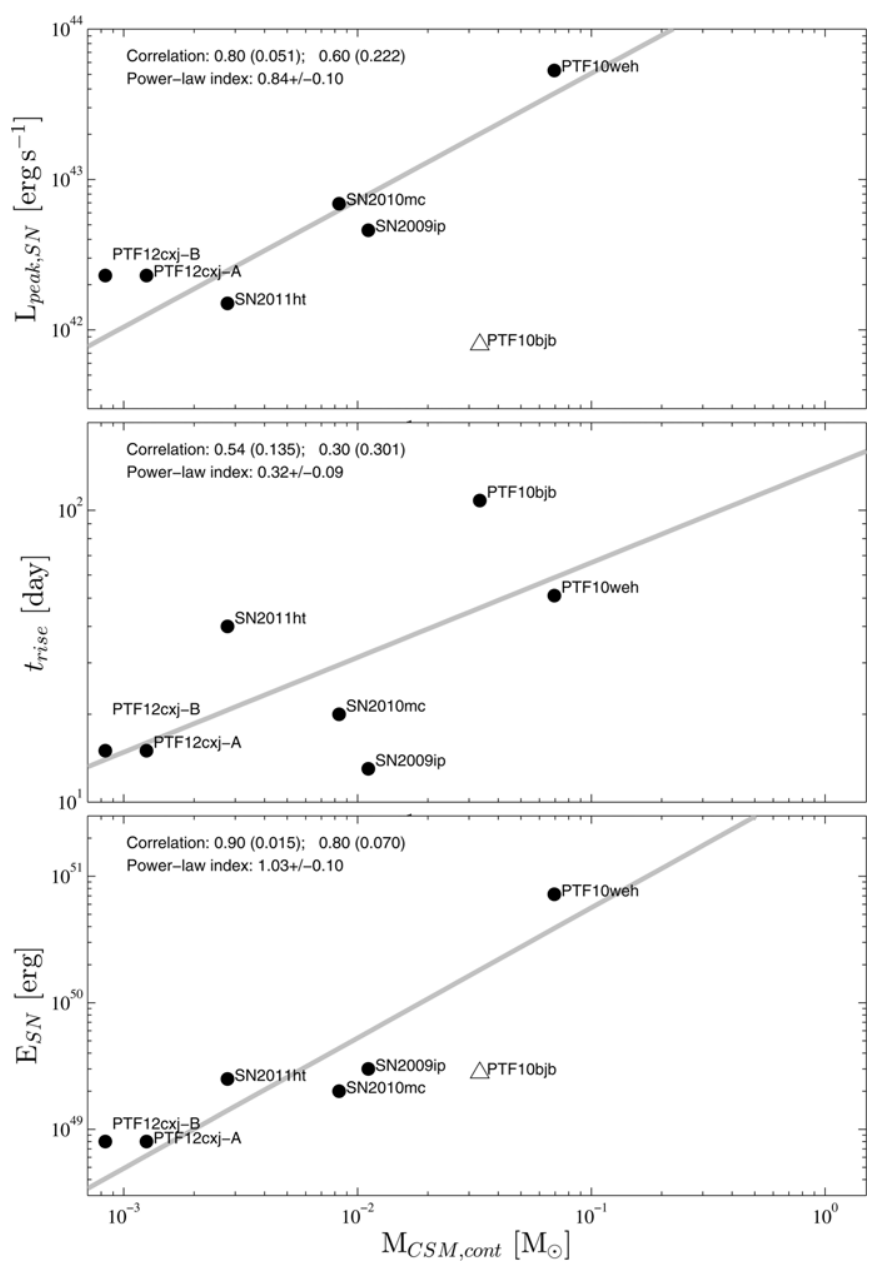

Figure 20. As in Figure 19, but with the CSM mass estimated based on Equation (5).

the Spearman rank correlation coefficient and the probability of getting this correlation by chance. Figure 20 shows the same, but for the CSM mass estimated based on Equation (5).

The clearest possible correlations we find are between (1) CSM mass estimated from Equation (4) and the SN peak luminosity; and (2) CSM mass estimated from Equation (5) and the total SN radiated energy. The false-alarm probabilities for these correlations are lower than $2 \%$. However, these correlations are based on a small sample of objects whose properties were measured only roughly. Therefore, a confirmation of this correlation requires a larger sample for which the properties of both $\mathrm{SNe}$ and precursors are accurately measured.

At this time, our observations cannot distinguish between scenarios in which the radiation drives a vigorous wind or an explosion drives a mass ejection. Nevertheless, these correlations may imply that at least the early-time optical light curves of SNe IIn are powered by shock breakout in an optically thick CSM followed by interaction of the SN ejecta with the CSM (Ofek et al. 2010; Chevalier \& Irwin 2011). The correlations we find between the $\mathrm{SN}$ and precursor properties also suggest that precursors are indeed accompanied by mass-loss events larger than $\sim 10^{-2} M_{\odot}$ (Figure 19). We note that we cannot rule out the possibility that these correlation, if real, arise from a different physical mechanism. Finally, we caution that given the small size of the sample, the reality of these correlations requires a verification based on a larger and better sample. 


\section{DISCUSSION}

We present the first systematic search for precursors prior to the explosion of Type IIn SNe. Our search yields five SNe for which at least one precursor is detected. Based on the sample control time, we are able to estimate the precursor rate. Our observations suggest that the rate of precursors brighter than an absolute magnitude of -14 in the last year prior to the SN IIn explosion is $\gtrsim 1 \mathrm{yr}^{-1}$. However, an important caveat is that some of our putative SN explosions might not actually be terminal events in which the star ends its life. Indeed, for the case of SN 2009ip, there have been some suggestions that the latest detected outburst is not the final SN explosion (e.g., Pastorello et al. 2013; however, see Mauerhan et al. 2014 for arguments to the contrary). We note that it will be possible to test this hypothesis using Hubble Space Telescope imaging by checking if the progenitor is still visible.

Next, we discuss some of the implications of this survey, assuming that the $\mathrm{SNe}$ in our sample do indeed represent the terminal explosion of their progenitors. Observations of SN 2010mc (Ofek et al. 2013b) suggest that, given the short period between the precursor and the explosion of SN 2010mc, the two events are probably causally connected, in the sense that the precursor is not random but instead deeply related to the late stages of stellar evolution. Following Ofek et al. (2013b), the remarkable rate of these precursors can be used to relate their nature to physical processes taking place during the final stages of stellar evolution. In the context of a model in which the precursors are associated with mass-loss events, ${ }^{14}$ we assume that each outburst releases on the order of $10^{-2} M_{\odot}$ (see Section 8; Ofek et al. 2013b, 2013c). Further assuming that the mass of the progenitor is on the order of $50 \mathrm{M}_{\odot}$, no more than $\sim 5000$ outburst events can occur during the life of the star. For a progenitor lifetime of $\sim 10^{7} \mathrm{yr}$, if the outbursts occur regularly during the star's life, then the average time interval between outbursts would be less than $\sim 2000 \mathrm{yr}$.

However, two observations make this scenario unlikely. The first is that if every SN IIn has 5000 precursors, then the observed rate in nearby galaxies would be two orders of magnitude higher than the SN rate. Such a high rate of precursors (e.g., "SN impostor"; Van Dyk \& Matheson 2012) is not seen, so it is likely that the number of precursors per $\mathrm{SN}$ is much lower. The second is that our results suggest that on order unity of these progenitors have precursors within a year prior to explosion, which means that the outbursts are much more likely to be physically connected to a process occurring near the end of a star's life, and probably not more than $\sim 5000 \mathrm{yr}(1 / 2000$ of their lifetime) prior to the SN explosion. For massive stars this is related to processes that occur after the beginning of carbon burning (Woosley et al. 2002). Furthermore, if each progenitor generates only a few outbursts (rather than the strict upper limit of 5000), then the physical process that is responsible for these outbursts likely takes place after the beginning of neon or oxygen burning (a few months to a few years prior to the explosion). The sole point of this rough estimate is to demonstrate that, in the context of this model, the precursors are likely related to some events taking place during the final stages of stellar evolution (rather than occurring at random times during the life of the star).

\footnotetext{
14 Unless the magnetic field is extremely high, we are not aware of any physical mechanism that can extract $\gtrsim 10$ Eddington luminosities without being accompanied by mass loss.
}

Finally, we note that mass-loss events are likely not limited to SN IIn progenitors; there is some evidence that other kinds of $\mathrm{SNe}$ have precursors prior to the explosion. For example, Corsi et al. (2014) reported on a possible eruption of the Type Ic SN PTF 11qcj about 2 yr prior to the SN explosion. Gal-Yam et al. (2014) and O. Yaron et al. (in preparation) show that spectra of some SNe obtained hours after the explosion reveal narrow, high-excitation emission lines that likely originate from a CSM that was ejected months to years prior to the SN explosion. The main difference is probably that in SNe IIn, the ejected mass is larger than in other classes of events.

E.O.O. thanks Orly Gnat and Ehud Nakar for many discussions. This paper is based on observations obtained with the Samuel Oschin Telescope as part of the Palomar Transient Factory project, a scientific collaboration between the California Institute of Technology, Columbia University, Las Cumbres Observatory, the Lawrence Berkeley National Laboratory, the National Energy Research Scientific Computing Center, the University of Oxford, and the Weizmann Institute of Science. Some of the data presented herein were obtained at the W. M. Keck Observatory, which is operated as a scientific partnership among the California Institute of Technology, the University of California, and NASA; the Observatory was made possible by the generous financial support of the W. M. Keck Foundation. We are grateful for excellent staff assistance at Palomar, Lick, and Keck Observatories. E.O.O. is incumbent of the Arye Dissentshik career development chair and is grateful for support by a grant from the Israeli Ministry of Science, ISF, Minerva, Weizmann-UK and the I-CORE Program of the Planning and Budgeting Committee and The Israel Science Foundation (grant no 1829/12). A.V.F.'s group at UC Berkeley has received generous financial assistance from Gary and Cynthia Bengier, the Christopher R. Redlich Fund, the Richard and Rhoda Goldman Fund, the TABASGO Foundation, and NSF grant AST-1211916.

\section{REFERENCES}

Arcavi, I., Gal-Yam, A., Yaron, O., et al. 2011, ApJL, 742, L18 Arnett, W. D., \& Meakin, C. 2011, ApJ, 741, 33

Boles, T., Pastorello, A., Stanishev, V., et al. 2011, CBET, 2851, 1

Challis, P., Foley, R. J., \& Berlind, P. 2010, CBET, 2243, 1

Chandra, P., Chevalier, R. A., Irwin, C. M., et al. 2012, ApJL, 750, L2 Chevalier, R. A. 2012, ApJL, 752, L2

Chevalier, R. A., \& Fransson, C. 1994, ApJ, 420, 268

Chevalier, R. A., \& Irwin, C. M. 2011, ApJL, 729, L6

Chevalier, R. A., \& Irwin, C. M. 2012, ApJL, 747, L17

Chugai, N. N., Cumming, R. J., Blinnikov, S. I., et al. 2003, arXiv: astro-ph/0309226

Chugai, N. N., \& Danziger, I. J. 1994, MNRAS, 268, 173

Ciabattari, F., Mazzoni, E., Koff, R. A., et al. 2011, CBET, 2830, 1

Corsi, A., Ofek, E. O., Gal-Yam, A., et al. 2014, ApJ, 782, 42

Dessart, L., Hillier, D. J., Gezari, S., Basa, S., \& Matheson, T. 2009, MNRAS, 394,21

Dintinjana, B., Mikuz, H., Skvarc, J., et al. 2011, CBET, 2906, 1

Drake, A. J., Djorgovski, S. G., Mahabal, A., et al. 2009, ApJ, 696, 870

Drake, A. J., Prieto, J. L., Djorgovski, S. G., et al. 2010, ATel, 2897, 1

Duszanowicz, G. 2010, CBET, 2241, 1

Edelson, R. A., \& Krolik, J. H. 1988, ApJ, 333, 646

Efron, B. 1982, The Jackknife, the Bootstrap and Other Resampling Plans (Philadelphia, PA: Society for Industrial and Applied Mathematics)

Filippenko, A. V. 1997, ARA\&A, 35, 309

Filippenko, A. V., Li, W. D., Treffers, R. R., \& Modjaz, M. 2001, in ASP Conf. Ser. 246, Small-Telescope Astronomy on Global Scales, ed. W. P. Chen, C. Lemme, \& B. Paczyński (San Francisco, CA: ASP), 121

Foley, R. J., Berger, E., Fox, O., et al. 2011, ApJ, 732, 32

Foley, R. J., Smith, N., Ganeshalingam, M., et al. 2007, ApJL, 657, L105

Fraser, M., Magee, M., Kotak, R., et al. 2013, ApJL, 779, L8

Gal-Yam, A., Arcavi, I., Ofek, E. O., et al. 2014, Natur, 509, 471 
Gal-Yam, A., \& Leonard, D. C. 2009, Natur, 458, 865

Gehrels, N. 1986, ApJ, 303, 336

Jin, Z., Gao, X., Brimacombe, J., Luppi, F., \& Buzzi, L. 2012, CBET, 3044, 1

Kiewe, M., Gal-Yam, A., Arcavi, I., et al. 2012, ApJ, 744, 10

Laher, R. R., Surace, J., Grillmair, C. J., et al. 2014, arXiv:1404.1953

Law, N. M., Kulkarni, S. R., Dekany, R. G., et al. 2009, PASP, 121, 1395

Li, W. D., Filippenko, A. V., Treffers, R. R., et al. 2000, in Cosmic Explosions, ed. S. S. Holt \& W. W. Zhang (New York: AIP), 103

Lupton, R. H., Gunn, J. E., \& Szalay, A. S. 1999, AJ, 118 , 1406

Mahabal, A., Drake, A. J., Djorgovski, S. G., et al. 2011, CBET, 2941, 1

Margutti, R., Milisavljevic, D., Soderberg, A. M., et al. 2014, ApJ, 780, 21

Mason, M., Cenko, S. B., Li, W., et al. 2011, CBET, 2712, 1

Mauerhan, J. C., Smith, N., Filippenko, A. V., et al. 2013a, MNRAS, 430, 1801

Mauerhan, J. C., Smith, N., Silverman, J. M., et al. 2013b, MNRAS, 431, 2599

Mauerhan, J. C., Williams, G. G., Smith, N., et al. 2014, MNRAS, in press (arXiv:1403.4240)

Moriya, T. J., Maeda, K., Taddia, F., et al. 2014, MNRAS, 439, 2917

Newton, J., \& Puckett, T. 2010, CBET, 2532, 1

Ofek, E. O., Arcavi, I., Tal, D., et al. 2014a, ApJ, 788, 154

Ofek, E. O., Cameron, P. B., Kasliwal, M. M., et al. 2007, ApJL, 659, L13

Ofek, E. O., Fox, D., Cenko, S. B., et al. 2013a, ApJ, 763, 42

Ofek, E. O., Laher, R., Law, N., et al. 2012a, PASP, 124, 62

Ofek, E. O., Laher, R., Surace, J., et al. 2012b, PASP, 124, 854

Ofek, E. O., Lin, L., Kouveliotou, C., et al. 2013c, ApJ, 768, 47

Ofek, E. O., Rabinak, I., Neill, J. D., et al. 2010, ApJ, 724, 1396

Ofek, E. O., Sullivan, M., Cenko, S. B., et al. 2013b, Natur, 494, 65

Ofek, E. O., Zoglauer, A., Boggs, S. E., et al. 2014b, ApJ, 781, 42

Ofek, O. 2012, CBET, 3313, 1

Pastorello, A., Cappellaro, E., Inserra, C., et al. 2013, ApJ, 767, 1

Pastorello, A., Mattila, S., Zampieri, L., et al. 2008, MNRAS, 389, 113

Pastorello, A., Smartt, S. J., Mattila, S., et al. 2007, Natur, 447, 829

Pastorello, A., Stanishev, V., Smartt, S. J., Fraser, M., \& Lindborg, M. 2011, CBET, 2851, 2
Prieto, J. L., Brimacombe, J., Drake, A. J., \& Howerton, S. 2013, ApJL 763, L27

Prieto, J. L., McMillan, R., Bakos, G., \& Grennan, D. 2011, CBET, 2903, 1

Quataert, E., \& Shiode, J. 2012, MNRAS, 423, L92

Rakavy, G., Shaviv, G., \& Zinamon, Z. 1967, ApJ, 150, 131

Rau, A., Kulkarni, S. R., Law, N. M., et al. 2009, PASP, 121, 1334

Rich, D. 2010, CBET, 2530, 1

Roming, P. W. A., Pritchard, T. A., Prieto, J. L., et al. 2012, ApJ, 751, 92

Schlegel, E. M. 1990, MNRAS, 244, 269

Shaviv, N. J. 2000, ApJL, 532, L137

Shaviv, N. J. 2001, MNRAS, 326, 126

Shiode, J. H., \& Quataert, E. 2014, ApJ, 780, 96

Silverman, J. M., Filippenko, A. V., \& Foley, R. J. 2010, CBET, 2538, 1

Smith, N., Chornock, R., Li, W., et al. 2008, ApJ, 686, 467

Smith, N., Li, W., Miller, A. A., et al. 2011, ApJ, 732, 63

Smith, N., Mauerhan, J. C., Silverman, J. M., et al. 2012, MNRAS, 426, 1905

Smith, N., Miller, A., Li, W., et al. 2010, AJ, 139, 1451

Smith, N., \& Owocki, S. P. 2006, ApJL, 645, L45

Smith, N., Silverman, J. M., Chornock, R., et al. 2009, ApJ, 695, 1334

Soker, N., \& Kashi, A. 2013, ApJL, 764, L6

Stoll, R., Prieto, J. L., Stanek, K. Z., et al. 2011, ApJ, 730, 34

Svirski, G., Nakar, E., \& Sari, R. 2012, ApJ, 759, 108

Szczygieł, D. M., Gerke, J. R., Kochanek, C. S., \& Stanek, K. Z. 2012, ApJ, 747,23

Valenti, S., Pastorello, A., Benetti, S., et al. 2011, CBET, 2906, 2

Van Dyk, S. D., \& Matheson, T. 2012, Eta Carinae and the Supernova Impostors, ed. K. Davidson \& R. M. Humphreys (Astrophysics and Space Science Library, Vol. 384; Dordrecht: Kluwer), 249

Woosley, S. E., Blinnikov, S., \& Heger, A. 2007, Natur, 450, 390

Woosley, S. E., Heger, A., \& Weaver, T. A. 2002, RvMP, 74, 1015

Yaron, O., \& Gal-Yam, A. 2012, PASP, 124, 668

Yoon, S.-C., \& Cantiello, M. 2010, ApJL, 717, L62

Zhang, T., Wang, X., Wu, C., et al. 2012, AJ, 144, 131 